TRANSACTIONS OF THE

AMERICAN MATHEMATICAL SOCIETY

Volume 350, Number 9, September 1998, Pages 3673-3706

S 0002-9947(98)01954-0

\title{
SELF-DUALITY OPERATORS ON ODD DIMENSIONAL MANIFOLDS
}

\author{
HOUHONG FAN
}

\begin{abstract}
In this paper we construct a new elliptic operator associated to any nowhere zero vector field on an odd-dimensional manifold and study its index theory. It turns out this operator has several geometric applications to conformal vector fields, self-dual vector fields, locally free $S^{1}$-actions and transversal hypersurfaces of these vector fields in an odd-dimensional manifold. In particular, we reveal a non-stable phenomena about the existence of conformal vector fields and self-dual vector fields in odd dimensions above 3. This is in sharp contrast to the stable phenomena about the existence of nowhere zero vector fields in odd dimensions. Besides these applications, the index formula of this new operator also gives the formulas for the dimensions of self-duality cohomology groups and for the virtual dimensions of the moduli spaces of anti-self-dual connections on 5-cobordisms, which are introduced in author's previous papers.
\end{abstract}

\section{INTRODUCTION}

It is well-known that topology of smooth 4-manifolds is more complicated than topology of higher dimensional smooth manifolds. However the topology of a smooth 4-manifold often can be captured by the topology of a line field on a much simpler smooth 5 -manifold. For example, any exotic 4 -space can be naturally represented by a smooth line field on the standard Euclidean 5 -space $\mathbb{R}^{5}$ (Arnold [1]). (An exotic 4-space is a smooth 4-manifold which is homeomorphic but not diffeomorphic to the standard Euclidean 4 -space $\mathbb{R}^{4}$.) This idea can also be applied to closed smooth 4-manifolds. In [12] we observe that such a representation of an exotic 4-space by a smooth line field is "faithful", which means that if there is a diffeomorphism of $\mathbb{R}^{5}$ sending a representative line field of one exotic 4-space to a representative line field of another exotic 4-space, then two exotic 4-spaces must be diffeomorphic. This observation motivates us to make the following definition. A smooth 4-space is a pair $(M, L)$ where $L$ is a smooth line field on a smooth 5manifold $M$. Two smooth 4 -spaces $(M, L)$ and $\left(M^{\prime}, L^{\prime}\right)$ are diffeomorphic if there is a diffeomorphism from $M$ onto $M^{\prime}$ taking $L$ to $L^{\prime}$. By this notion it is natural to ask how to extend theories on smooth 4-manifolds to smooth 4-spaces. This suggests many interesting research topics. In addition it is perhaps more appropriate

Received by the editors August 18, 1995 and, in revised form, October 6, 1996 .

1991 Mathematics Subject Classification. Primary 57R25, 58G10; Secondary 57R80, 57M99, $58 \mathrm{~F} 25$.

(C) 1998 American Mathematical Society 
to study deformations of smooth structures of smooth 4-spaces rather than those of smooth 4-manifolds alone.

The gauge theory on smooth 4-manifolds is one of the most profound theories. How can the gauge theory on smooth 4-manifolds be extended to smooth 4-spaces? In [11] and [12] we proposed the self-dual Yang-Mills-type gauge theory and its dual Seiberg-Witten-type theory on smooth 5-manifolds with a smooth line field, that is, smooth 4-spaces. The linear models of these two non-linear theories are essentially a new operator which we are going to construct and study in this paper. We call this operator the self-duality operator, for it can be regarded as an analogue of the self-duality operator in the gauge theory on smooth 4-manifolds. Since we find our construction of the self-duality operator works quite well in any odd dimension $\geq 3$, we are not going to restrict our studies only to dimension 5 in this paper.

To make the reading of this paper more pleasant, in Section 1 we discuss many applications of the self-duality operators to self-dual vector fields, nearly-self-dual vector fields and conformal vector fields. We first recall the notion of a self-dual vector field from [10] and generalize this to the notion of a nearly-self-dual vector field (see Definitions 1.1 and 5.1). Any conformal vector field on an odd-dimensional manifold is self-dual. Then we state a main theorem about the non-stable phenomena for the existence of nearly-self-dual vector fields in odd dimensions above 3, which is in sharp contrast to the stable phenomena for the existence of nowhere zero vector fields in odd dimensions (see Theorems 1.5 and 1.7 and Proposition 1.6). This result follows from the necessary conditions we find for the existence of nearlyself-dual vector fields on an odd-dimensional manifold (see Theorems 1.10, 1.11 and 1.15). In addition we obtain several topological inequalities about transversal hypersurfaces of nearly-self-dual vector fields (see Theorems 1.16, 1.17 and 5.7). The proofs of all these results are presented in the final section.

In Section 2 we construct the self-duality operator associated to a line field on an odd-dimensional manifold (see Definition 2.3). Then we state the index formula for the self-duality operator with an elliptic boundary condition when the manifold is compact with boundary and the line field is transverse to the boundary (see Theorem 2.9). The proof of this formula is postponed to the final section. This index formula has several other significant applications besides those discussed in Section 1. It yields the formulas for the virtual dimension of the moduli space of anti-self-dual connections on a 5-cobordism in [11], and the virtual dimension of the moduli space of Seiberg-Witten-type monopoles on a 5-cobordism in [12] (see Corollaries 2.11 and 2.12). In Section 3, we give an application of the index formula to a general nowhere zero vector field (see Corollary 3.1).

In Section 4 we define the self-duality cohomology groups associated to a general line field on a compact odd-dimensional manifold and get many topological inequalities about the dimensions of these self-duality cohomology groups. In Section 5 we focus our attention to nearly-self-dual vector fields again. By using the index formula of self-duality operator we get formulas for all the dimensions of the self-duality cohomology groups associated to a nearly-self-dual vector field (see Theorem 5.6). In the final section we present all the postponed proofs.

We want to emphasize that the line fields in all sections except Sections 1 and 5 are general ones. Sections 1 and 5 concern the applications of self-duality operators to nearly-self-dual line fields. Finally note that this paper is the published version of the author's preprint "Self-dual vector fields on odd dimensional manifolds". 


\section{Applications to NEARLY-SELF-DUAL Line FIELdS I}

In this section we first discuss the applications of self-duality operators to nearlyself-dual line fields. Let $L$ be a smooth tangent line field on a smooth $(2 m+1)$ manifold $M$. Define

$$
Q^{*} \stackrel{\text { def }}{=}\left\{\omega \in T^{*} M \mid\langle\omega, L\rangle=0\right\} .
$$

Then $Q^{*}$ is a real $2 m$-dimensional subbundle of $T^{*} M$. A fiberwise Riemannian metric $g_{q}$ on $Q^{*}$ and an orientation on $Q^{*}$ determine an operator

$$
*_{q}: \bigwedge^{j} Q^{2 m-j} \bigwedge^{*} Q^{*}
$$

by $\alpha \wedge *_{q} \beta=g_{q}(\alpha, \beta) \operatorname{vol}\left(g_{q}\right)$ for any $\alpha, \beta \in \bigwedge^{j} Q^{*}$, where $\operatorname{vol}\left(g_{q}\right)$ is the oriented volume element of $g_{q}$.

Let $L_{V}$ denote the Lie derivative with respect to a vector $V$. For any section $V$ of the line field $L$, define

$$
\Gamma_{V} \stackrel{\text { def }}{=} L_{V} *_{q}-*_{q} L_{V}: \bigwedge^{m} Q^{*} \rightarrow \bigwedge^{m} Q^{*}
$$

The following definition is a special case of ([10], Definition 6.6).

Definition 1.1. A tangent line field $L$ on a smooth $(2 m+1)$-manifold $M$ is called self-dual if it is smooth and there is a smooth fiberwise Riemannian metric $g_{q}$ on $Q^{*}$ and an orientation on $Q^{*}$ such that $\Gamma_{V} \stackrel{\text { def }}{=} L_{V} *_{q}-*_{q} L_{V}=0$ on sections of $\bigwedge^{m} Q^{*}$ for any smooth section $V$ of $L$. Such a metric $g_{q}$ on $Q^{*}$ is called $L$-compatible. A vector field is called self-dual if it is smooth, nowhere zero and the line field generated by the vector field is self-dual.

The vanishing of $\Gamma_{V}$ means that the operator $*_{q}$ commutes with the Lie derivative $L_{V}$, that is, all self-dual sections of $\bigwedge^{m} Q^{*}$ are preserved by the flow generated by $V$. This is the reason why we call such a line field self-dual. Note that $\Gamma_{V}$ is a zero-th order operator on $M$, that is, $\Gamma_{V}(f \omega)=f \Gamma_{V} \omega$ for any smooth function $f$ and any smooth section $\omega$ of $\bigwedge^{m} Q^{*}$ over $M$. In addition $\Gamma_{f V}=f \Gamma_{V}$ on $\bigwedge^{m} Q^{*}$ for any function $f$ and section $V$ of $L$ over $M$.

In fact what we need is a notion of a nearly-self-dual vector field, which requires the commutativity of $*_{q}$ and $L_{V}$ only on certain smooth sections of $\bigwedge^{m} Q^{*}$. But the definition technically gets more involved, so we defer it to Definition 5.1. For this section we only need to know

Lemma 1.2. (1) Any self-dual line field is nearly self-dual.

(2) If a vector field $V$ is self-dual (nearly-self-dual resp.) over an open subset $O$ then $f V$ is self-dual (nearly-self-dual resp.) over $O$ for any nowhere zero smooth function $f$ over $O$.

The first assertion is obvious by Definitions 1.1 and 5.1. The second assertion follows from the fact that $V$ and $f V$ generate the same line field over $O$. Recall that a vector field $V$ is conformal if $V$ is nowhere zero, smooth and the 1-dimensional foliation generated by $V$ is conformal. Any Riemannian vector field is conformal. The vector field induced by a locally free smooth $S^{1}$-action is Riemannian. (See Vaisman [21] and Appendix A by Y. Carriere in Molino [17] or Section 6 of [10].)

Lemma 1.3. If a vector field on an odd-dimensional orientable manifold is conformal, then it is self-dual. 
For the proof of this fact see ([10], Remark 6.8). For a $(2 m+1)$-manifold $M$ and a subspace $K \subset M$, the half-characteristic and relative half-characteristic are respectively defined as

$$
\chi_{\frac{1}{2}}(M)=\sum_{j=0}^{m-1}(-1)^{j} b^{j}(M), \quad \chi_{\frac{1}{2}}(M, K)=\sum_{j=0}^{m-1}(-1)^{j} b^{j}(M, K) .
$$

Here $b^{j}(M)$ denotes the $j$-th Betti number of $M$ and $b^{j}(M, K)$ denotes the $j$-th relative Betti number of $(M, K)$. Note that $\chi_{\frac{1}{2}}$ is different from the Kervaire semicharacteristic.

Definition 1.4. A vector field on a smooth compact manifold with boundary is called proper if it is smooth, nowhere zero and transverse to the boundary.

Now we can state a main result in this paper.

Theorem 1.5. Let $M$ be a compact, connected oriented smooth $(2 m+1)$-manifold with nonempty boundary $\partial M$ and $\operatorname{dim} M \geq 5$. Let $Y$ be a closed connected oriented smooth $(2 m+1)$-manifold such that $\chi_{\frac{1}{2}}(Y)<1$ in the case $m$ is odd and $\chi_{\frac{1}{2}}(Y)>1$ in the case $m$ is even. Then for all sufficiently large $k, M \sharp k Y$ doesn’t admit any nearly-self-dual proper vector field.

This theorem should be compared with the following result.

Proposition 1.6. Let $M$ be a compact, connected oriented smooth $(2 m+1)$ manifold with nonempty boundary $\partial M$, and let $N$ be the union of some boundary components of $M(N$ may be empty). Suppose that $\chi(N)=\chi(\partial M-N)$. Then for any given closed oriented smooth $(2 m+1)$-manifold $Y$, the connected sum $M \sharp Y$ always admits a proper vector field which is outward on $N$ and inward on $\partial M-N$.

Thus in odd dimensions the existence of a proper vector field is a stable phenomena. But by contrast, Theorem 1.5 reveals that in all odd dimensions $\geq 5$ the existence of a proper nearly-self-dual vector field is a non-stable phenomena.

Theorem 1.5 follows from Theorems 1.10 and 1.11 and Lemma 1.8. Proposition 1.6 follows from the fact that the condition $\chi(N)=\chi(\partial M-N)$ in Theorem 1.12 depends only on the boundary. Theorem 1.5 also can be applied to a closed odddimensional manifold.

Theorem 1.7. Let $M$ be a closed, connected oriented smooth $(2 m+1)$-manifold of $\operatorname{dim} M \geq 5$. Let $Y$ be as in Theorem 1.5. Then for all sufficiently large $k, M \sharp k Y$ doesn't admit any nearly-self-dual vector field which is transverse to a smoothly imbedded closed $2 m$-manifold $C$ in $M$, and in particular, $M \sharp k Y$ doesn’t admit any locally free smooth $S^{1}$-action which is transverse to a smoothly imbedded closed $2 m$-manifold $C$ in $M$.

Indeed if such a nearly-self-dual vector field existed, then we could cut $M$ along $C$ and got a situation in contradiction to Theorem 1.5. (A locally free smooth $S^{1}$-action transverse to $C$ just means that the vector field induced by it is so.)

It should be emphasized that there is a qualitative difference between the invariants $\chi_{\frac{1}{2}}(M, K)$ and $\chi(M, K)$. For an odd-dimensional compact oriented smooth manifold $M$ with boundary $\partial M$ and $K \subset \partial M$,

$$
\chi(M, K)=\frac{1}{2} \chi(\partial M)-\chi(K),
$$


which depends only on the boundary $\partial M$. Thus $\chi(M, K)$ remains unchanged when $M$ is replaced by $M \sharp k Y$ for any closed oriented manifold $Y$ of the same dimension. However, $\chi_{\frac{1}{2}}(M, K)$ is changed in general if we do so in odd dimensions $\geq 5$.

Lemma 1.8. Let $M$ be a compact connected oriented $(2 m+1)$-manifold. Let $Y$ be a closed connected oriented $(2 m+1)$-manifold. Then

$$
\chi_{\frac{1}{2}}(M \sharp k Y)=\chi_{\frac{1}{2}}(M)+k\left(\chi_{\frac{1}{2}}(Y)-1\right) .
$$

Examples of $Y$ with $\chi_{\frac{1}{2}}(Y) \neq 1$ :

(1) Let $\Sigma^{n}$ denote an oriented rational homology $n$-sphere, that is, a closed oriented smooth $n$-manifold such that $H_{j}\left(\Sigma^{n}, \mathbb{Q}\right)=H_{j}\left(S^{n}, \mathbb{Q}\right)$ for all $j$. Some examples are $n$-spheres, exotic $n$-spheres, odd-dimensional real projective spaces, lens spaces, oriented quotients of any homotopy $n$-sphere by a free action of a finite group. For any $2 m+1 \geq 7,1 \leq j \leq m-1$, we have

$$
\chi_{\frac{1}{2}}\left(\Sigma^{j} \times \Sigma^{2 m-j+1}\right)=1+(-1)^{j} .
$$

Note that $S^{j} \times S^{2 m-j+1}$ for $j \geq 2$ is simply-connected. So the operation of the connected sum with $S^{j} \times S^{2 m-j+1}$ for $j \geq 2$ preserves the fundamental group.

(2) For any $2 m$-manifold $V$ we have

$$
\chi_{\frac{1}{2}}\left(S^{1} \times V\right)=(-1)^{m-1} b^{m-1}(V) .
$$

Thus $\chi_{\frac{1}{2}}\left(S^{1} \times V\right) \neq 1$ unless $m$ is odd and $b^{m-1}(V)=1$. For example, for $2 m+1 \geq 5$ and $4 k+1 \geq 5$, we have

$$
\chi_{\frac{1}{2}}\left(S^{1} \times \Sigma^{2 m}\right)=\chi_{\frac{1}{2}}\left(S^{1} \times \mathbb{C} P^{2 k}\right)=0 .
$$

Now we state the following main theorem about necessary conditions for the existence of a nearly-self-dual proper vector field. It will be proved in the final section by using self-duality cohomology groups. Let $N^{c} \stackrel{\text { def }}{=} \partial M-N$ and $K^{c} \stackrel{\text { def }}{=}$ $\partial M-K$.

Theorem 1.9. Let $M$ be a compact connected oriented smooth $(2 m+1)$-manifold $M$ with nonempty boundary and $N$ be the union of some components of $\partial M$. Suppose $M$ admits a nearly-self-dual proper vector field outward on $N$ and inward on $\partial M-N$. Then

$$
(-1)^{m} \chi_{\frac{1}{2}}(M, K) \leq \frac{(-1)^{m}}{4}[\chi(\partial M)-2 \chi(K)]-\frac{1}{2}\left|\sigma(N \cap K)+\sigma\left(N^{c} \cap K^{c}\right)\right| .
$$

for any union $K$ of components of $\partial M$ such that $K \neq \partial M$. Here the signature $\sigma$ is with respect to the outward orientation. In particular, we have

$$
(-1)^{m} \chi_{\frac{1}{2}}(M) \leq \frac{(-1)^{m}}{4} \chi(\partial M)-\frac{1}{2}|\sigma(N)| .
$$

For convenience in applications, we restate Theorem 1.9 separately in the case of odd $m$ and in the case of even $m$.

Theorem 1.10. Let $M$ be a compact connected orientable smooth (4k+3)-manifold $M$ with nonempty boundary and with integer $k \geq 0$. Suppose $M$ admits a nearlyself-dual proper vector field $V$. Then

$$
\chi_{\frac{1}{2}}(M, K) \geq \frac{1}{4} \chi(\partial M)-\frac{1}{2} \chi(K)
$$


for any union $K$ of boundary components of $M$ such that $K \neq \partial M$. In particular,

$$
\chi_{\frac{1}{2}}(M) \geq \frac{1}{4} \chi(\partial M) .
$$

Theorem 1.11. Let $M$ be a compact connected oriented smooth $(4 k+1)$-manifold $M$ with nonempty boundary and with integer $k \geq 1$. Suppose $M$ admits a nearlyself-dual proper vector field $V$ which is outward on $N$ and inward on $\partial M-N$, where $N$ is a union of some boundary components of $M$ ( $N$ may be empty). Then

$$
\chi_{\frac{1}{2}}(M, K) \leq \frac{1}{4} \chi(\partial M)-\frac{1}{2} \chi(K)-\frac{1}{2}\left|\sigma(N \cap K)+\sigma\left(N^{c} \cap K^{c}\right)\right|
$$

for any union $K$ of boundary components of $M$ such that $K \neq \partial M$. In particular,

$$
\chi_{\frac{1}{2}}(M) \leq \frac{1}{4} \chi(\partial M)-\frac{1}{2}|\sigma(N)|
$$

Note that the right sides of the inequalities in Theorems 1.9, 1.10 and 1.11 depend only on the boundary $\partial M$, while the left sides depend on the whole manifold $M$.

To make comparisons, we recall the classical Hopf Theorem (in its simplest version) states that there is a nowhere zero smooth vector field on a closed smooth manifold $M$ if and only if $\chi(M)=0$. The following is the relative version of this theorem.

Theorem 1.12 (Relative Hopf Theorem). Let $M$ be a compact connected oriented smooth n-manifold with boundary $\partial M$. Let $N$ be the union of some boundary components of $M$ ( $N$ may be empty). Then there is a proper vector field which is outward on $N$ and inward on $\partial M-N$ if and only if $\chi(M, N)=0$. In the case $\operatorname{dim} M$ is odd, $\chi(M, N)=0$ is equivalent to $\chi(N)=\chi(\partial M-N)$.

This relative version can be proved in the same way as the original Hopf Theorem on closed smooth manifolds. (1) The sum of indices of isolated singularities of vector fields outward on $N$ and inward on $\partial M-N$ is independent of such vector fields. (This follows from the facts that the indices are degrees of the Gauss maps and degrees of maps are homotopy invariant.) (2) There is a Morse function on $M$ which is 0 on $N$ and 1 on $\partial M-N$. The index of the gradient vector field of the Morse function at a critical point is $(-1)^{\lambda}$ where $\lambda$ is the Morse index of the critical point. The sum of them is $\chi(M, N)$. For the converse statement, take any vector field which points outward on $N$ and inward on $\partial M-N$ and has only isolated singularities. First move all the isolated singularities into a coordinate chart by a one-parameter of diffeomorphisms which preserve a collar of $\partial M$. Secondly perturb the vector field so that all indices are either +1 or -1 . Thirdly join two singularities of degrees +1 and -1 by disjoint arcs. (In dimension 2 , use an easy surgery to achieve this.) Finally isotope the vector field near the arc to make both singularities disappear. For details of these steps see (Hirsch [14], pp. 136-137, p. 161).

The second statement in Theorem 1.12 follows from the fact that $\chi(M)=$ $\frac{1}{2} \chi(\partial M)$ for any odd-dimensional compact oriented smooth manifold $M$ with boundary $\partial M$.

Remark 1.13. (1) If one regards a nearly-self-dual (or self-dual) vector field as a geometric structure in odd dimensions, just as one regards a nondegenerate closed 2 -form as a symplectic structure in even dimensions, then one may compare the non-stable phenomena of the existence of nearly-self-dual proper vector fields in 
odd dimensions $\geq 5$ with the non-stable phenomena in the differential topology in dimension 4. For example, the connected sum of two closed smooth 4-manifolds with $b_{+}^{2}>1$ doesn't admit any Kähler structure (a theorem of Donaldson, [9]). In fact, such a connected sum doesn't even admit any symplectic structure (a theorem of Taubes, [20]). These results in dimension 4 are proved by using the (anti-selfdual) Yang-Mills equation and the Seiberg-Witten equation, which linearizations are essentially the self-duality operator in dimension 4 (and the Dirac operator). Our proof for Theorem 1.5 is based on an analogue of the self-duality operator in odd dimensions. Of course, the Yang-Mills equation and Seiberg-Witten equation are much deeper than their linearized equations. On the other hand, the YangMills equation and Seiberg-Witten equation in dimension 4 have been generalized to dimension 5 in [11] and [12]. The consequences of these nonlinear equations on 5 -manifolds remain to be investigated further.

(2) For any contact structure on an odd-dimensional smooth manifold, there is a naturally associated vector field, which is called the characteristic vector field of the contact structure. The characteristic vector field of any K-contact structure is Riemannian. A special class of K-contact structures are so-called Sasakian structures, which are regarded as the odd-dimensional analogue of Kähler structures in even dimensions. Much work has been done about the relations between the underlying topology and Sasakian structures and K-contact structures. (See Blair [6], pp.64-80.) It is natural to hope these studies can be extended to self-dual contact structures, that is, the contact structures whose characteristic vector fields are self-dual.

(3) In the case $\left(M ; N_{0}, N_{1}\right)$ is an oriented compact smooth rational homology $h$-cobordism triad, i.e., $H_{j}\left(M, N_{i} ; \mathbb{Q}\right)=0$ for $i=0,1$ and all $j$, then all inequalities in Theorems 1.10 and 1.11 are satisfied with $N=N_{1}$. Indeed in such a case we have $b^{j}(M)=b^{j}\left(N_{1}\right)=b^{j}\left(N_{0}\right)$ for all $j$ and thus $\chi_{\frac{1}{2}}\left(M, N_{1}\right)=\chi_{\frac{1}{2}}\left(M, N_{0}\right)=$ 0 and $\chi\left(N_{1}\right)=\chi\left(N_{0}\right)=\frac{1}{2} \chi(\partial M)$. Moreover when $\operatorname{dim} M=4 k+3$, we have $\chi_{\frac{1}{2}}(M)=\frac{1}{4} \chi(\partial M)+\frac{1}{2} b^{2 k+1}(N)$. When $\operatorname{dim} M=4 k+1$, we have $\chi_{\frac{1}{2}}(M)=$ $\frac{1}{4} \chi(\partial M)-\frac{1}{2} b^{2 k}(N) \leq \frac{1}{4} \chi(\partial M)-\frac{1}{2}|\sigma(N)|$. Due to this fact, it is natural to ask

Question 1.14. Is there any obstruction for the existence of a nearly-self-dual proper vector field $V$ on a compact oriented smooth odd-dimensional $h$-cobordism such that $V$ is outward on one boundary component and inward on the other boundary component?

Of course, if $\left(M ; N_{0}, N_{1}\right)$ is an oriented compact smooth $s$-cobordism triad of dimension $>5$, then the $s$-cobordism Theorem implies that it is a smooth product, and therefore there is an obvious self-dual vector field as required. The most interesting cases are probably in dimensions 3 and 5 , for the relation of this problem to low-dimensional topology may turn out to be important.

When $\operatorname{dim} M=3, V$ being self-dual is equivalent to $V$ being conformal, and is also equivalent to the fact that $V$ is transversely holomorphic. Transversely holomorphic vector fields which are transverse to some codimension-one foliation on a closed oriented 3-manifold are classified by Brunella and Ghys [7].

In this paper we can deduce the following result about nearly-self-dual vector fields on 3-manifolds from Theorem 5.6. Its full proof will be presented in the final section. 
Theorem 1.15. Let $M$ be a compact connected orientable 3-manifold with $k>0$ many boundary components. If $k \neq 2$ and one of the boundary components of $M$ is not torus, then $M$ doesn't admit any nearly-self-dual proper vector field.

As an example of Theorem 1.15, a compact connected orientable 3-manifold $M$ with three boundary components $S^{2}, S^{2}$ and $T^{2}$ (a torus) always admits a proper vector field which is outward on one $S^{2}$ and inward on the other $S^{2}$ and $T^{2}$. But it never admits any nearly-self-dual proper vector field.

In dimension 4, the Yang-Mills equation and the Seiberg-Witten equation can be applied to get lower bounds for the genus of smoothly imbedded surfaces in smooth 4-manifolds. (Kronheimer and Mrowka [15], [16], Morgan, Szabo and Taubes [18].) The self-duality operator in odd-dimensions can also be applied to get lower bounds of middle-dimensional Betti numbers and $b_{ \pm}$-invariants of a smoothly imbedded codimension-one closed manifold which is transverse to a self-dual proper vector field.

Recall for any closed oriented $2 m$-manifold $K$ with even $m, b_{+}^{m}(K)\left(b_{-}^{m}(K)\right.$ resp. ) is the dimension of maximal positive ( negative resp. ) subspace for the intersection form on $H^{m}(K)$. For any closed oriented $2 m$-manifold $K$ with odd $m$, $b_{ \pm}^{m}(K) \stackrel{\text { def }}{=} \frac{1}{2} b^{m}(K)$. The following theorem follows immediately from Theorem 5.7, which will be proved by using self-duality cohomology groups. (See Remark 5.8.)

Theorem 1.16. Let $M$ be a compact connected oriented smooth $(2 m+1)$-manifold with nonempty boundary and with a nearly-self-dual proper vector field $V$ and integer $m \geq 1$. Let $C$ be a smoothly imbedded closed $2 m$-manifold in $M$ which is transverse to $V$, and let $C$ be so oriented that $V \wedge$ orientation of $C$ agrees with the orientation of $M$. Let $N$ denote the union of components where $V$ is outward. Suppose either (1) $C$ is a part of the boundary of $M$ or (2) $V$ is self-dual. Then we have

$$
b_{ \pm}^{m}(C) \geq(-1)^{m} \chi_{\frac{1}{2}}(M, \partial M)+\frac{(-1)^{m}}{4}[\chi(\partial M) \pm 2 \sigma(N)]
$$

Here $N$ and $\partial M$ takes the outward orientation induced from the orientation of $M$.

Theorems 1.9 and 1.16 can be applied to a closed odd-dimensional manifold. Recall for any closed oriented $2 m$-manifold $C$ we have

$$
\chi^{ \pm}(C) \stackrel{\text { def }}{=} \frac{1}{2}[\chi(C) \pm \sigma(C)]
$$

Let $-C$ denote $C$ with the opposite orientation.

Theorem 1.17. Let $M$ be a closed, connected oriented smooth $(2 m+1)$-manifold $M$ with a nearly-self-dual vector field $V$ and integer $m \geq 1$. Let $C$ be a smoothly imbedded connected closed $2 m$-manifold in $M$ which is transverse to $V$, and let $C$ be so oriented that $V \wedge$ orientation of $C$ agrees with the orientation of $M$.

(1) In the case $C$ doesn't separate $M$, we can cut $M$ along $C$ and get a manifold $\hat{M}$ such that $\partial \hat{M}=C \sqcup-C$. Then we have

$$
\begin{gathered}
(-1)^{m} \chi^{ \pm}(C) \geq(-1)^{m} \chi_{\frac{1}{2}}(\hat{M}), \\
0 \geq(-1)^{m} \chi_{\frac{1}{2}}(\hat{M}, C), \\
0 \geq(-1)^{m} \chi_{\frac{1}{2}}(\hat{M},-C),
\end{gathered}
$$




$$
b_{ \pm}^{m}(C)+\frac{(-1)^{m+1}}{2} \chi^{ \pm}(C) \geq(-1)^{m} \chi_{\frac{1}{2}}(\hat{M}, C \sqcup-C) .
$$

(2) In the case $C$ separates $M$, we can cut $M$ along $C$ and get a manifold $M_{1}$ with $\partial M_{1}=C$ where $V$ is outward, and another manifold $M_{2}$ with $\partial M_{2}=-C$ where $V$ is inward. Then for $i=1,2$, we have

$$
\begin{gathered}
\chi(C)=0, \quad \sigma(C)=0, \\
0 \geq(-1)^{m} \chi_{\frac{1}{2}}\left(M_{i}\right), \\
\frac{b^{m}(C)}{2} \geq(-1)^{m} \chi_{\frac{1}{2}}\left(M_{i}, C\right) .
\end{gathered}
$$

Proof. Inequality (1.18) follows from (1.11) applied to $\hat{M}$ with $N=C$ and (1.17). Inequality (1.19) ( (1.20) resp. ) follows from (1.10) applied to $\hat{M}$ with $N=C$ and $K=C$ ( $K=-C$ resp. ). Inequality (1.21) follows from (1.16) applied to $\hat{M}$ and (1.17).

For (1.22), $\chi(C)=0$ follows from Theorem 1.12. $\sigma(C)=0$ since $C$ bounds $M_{1}$. Inequality (1.23) follows from (1.11) applied to $M_{i}$ and (1.22) and (1.17). Inequality (1.24) follows from (1.16) applied to $M_{i}$ and (1.22), where we note that $0=\sigma(C)=b_{+}^{m}(C)-b_{-}^{m}(C)$ implies $b_{+}^{m}(C)=b_{-}^{m}(C)=\frac{b^{m}(C)}{2}$.

Note that the left sides of (1.18), (1.21) and (1.24) depend only on $C$, but the right sides of (1.18), (1.19), (1.20), (1.21), (1.23) and (1.24) depend on the whole $M$.

\section{The SELF-DUAlity OPERATOR OF A GENERAL LINE FIELD}

Let $L$ be any given smooth tangent line field on a smooth $(2 m+1)$-manifold $M$. Recall (1.1) for the definition of $Q^{*}$. It can be canonically identified as the dual of the intrinsic quotient bundle $T M / L$ in the following way. Let $[Y]$ denote the equivalent class of $Y$ in $T M / L$ for $Y \in T M$. Because the paring $\langle\omega,[Y]\rangle \stackrel{\text { def }}{=}\langle\omega, Y\rangle$ is well-defined for any $\omega \in Q^{*}$, it induces an identification $Q^{*}$ with $\operatorname{Hom}_{\mathbb{R}}(T M / L, \mathbb{R})$ in the obvious way. In the theory of foliations, one usually use the intrinsic quotient bundle. However, in this paper we would like to regard $Q^{*}$ as a subbundle of $T^{*} M$ so that the whole calculus of differential forms is at our disposal.

Definition 2.1. Let $M$ be an orientable smooth $(2 m+1)$-manifold. By the term data $\left(L, g, o_{q}\right)$ on $M$, we mean that $L$ is a smooth orientable tangent line field on $M, g$ is a smooth Riemannian metric on $M$, and $o_{q}$ is an orientation on the dual codimension-one cotangent plane field $Q^{*} \stackrel{\text { def }}{=} L^{\perp}$ over $M$, where $\perp$ is with respect to the paring of a vector and covector.

For a given data $\left(L, g, o_{q}\right)$, let $\left.g_{q} \stackrel{\text { def }}{=} g\right|_{Q^{*}}$, which is a smooth fiberwise Riemannian metric on $Q^{*}$. An orientation on $Q^{*}$ and a metric on $Q^{*}$ are called a quotient-orientation and a quotient-metric respectively.

Let $L^{*}$ be the $g$-orthogonal complement of $Q^{*}$ in $T^{*} M$. It is a cotangent line field. Let $\left.g_{l} \stackrel{\text { def }}{=} g\right|_{L^{*}}$, which is a smooth fiberwise Riemannian metric on $L^{*}$. Obviously we have $g_{l} \oplus g_{q}=g$ and the following orthogonal decomposition:

$$
T^{*} M=L^{*} \oplus Q^{*}
$$

A section of $\bigwedge^{j} Q^{*}$ is called a quotient $j$-form. 
Choose an orientation $o$ on $M$. Then the orientation $o_{l}$ on $L^{*}$ is defined by $o_{l} \wedge o_{q}=o$. It induces a unique orientation of $L$ by requiring that $\langle\nu, V\rangle>0$ for any positively oriented nowhere zero sections $\nu$ of $L^{*}$ and $V$ of $L$. This orientation on $L$ is still denoted as $o_{l}$.

For the sake of brevity we make the following convention throughout this paper.

Convention 2.2. $m$ is a positive integer $\geq 1$. When $m$ is odd, all forms are complex forms; $\Omega^{j}(M)$ is the space of sections of $\bigwedge^{j} T_{c}^{*} M$ where $T_{c}^{*} M \stackrel{\text { def }}{=} \mathbb{C} \otimes_{\mathbb{R}}$ $T^{*} M ; \Omega_{q}^{j}(M)$ is the space of sections of $\bigwedge^{j} Q_{c}^{*}$ where $Q_{c}^{*} \stackrel{\text { def }}{=} \mathbb{C} \otimes_{\mathbb{R}} Q^{*} ; E$ is a complex vector bundle over $M ; \Omega^{j}(E)$ is the space of sections of $\bigwedge^{j} T_{c}^{*} M \otimes_{\mathbb{C}} E ; \Omega_{q}^{j}(E)$ is the space of sections of $\bigwedge^{j} Q_{c}^{*} \otimes_{\mathbb{C}} E$; dim means complex dimensions; $H^{j}(M)$ means $H^{j}(M, \mathbb{C})$; and $s$ is a nonzero complex number.

When $m$ is even, all forms are real forms; $\Omega^{j}(M)$ is the space of sections of $\bigwedge^{j} T^{*} M ; \Omega_{q}^{j}(M)$ is the space of sections of $\bigwedge^{j} Q^{*} ; E$ is a real or complex vector bundle over $M ; \Omega^{j}(E)$ is the space of sections of $\bigwedge^{j} T^{*} M \otimes_{\mathbb{R}} E ; \Omega_{q}^{j}(E)$ is the space of sections of $\bigwedge^{j} Q^{*} \otimes_{\mathbb{R}} E ;$ dim means real dimensions; $H^{j}(M)$ means $H^{j}(M, \mathbb{R})$; and $s$ is a nonzero real or complex number according to the bundle $E$ is real or complex.

To make notations simpler, we omit mention of $E$ in the notations such as $\operatorname{dim} \mathcal{H}_{+}^{m}(A, K)$ when the bundle $E$ is fixed in our discussions. All forms and metrics in this paper are smooth, here the word "smooth" means class $C^{k}$ where $k$ is sufficiently large.

For those readers who have read [10], it should be reminded that the notations in this paper are different from those in [10]. The correspondence is as follows: $L, L^{*}$, $o_{l}, g_{l}, Q^{*}, o_{q}, g_{q}$ and $\Gamma_{V}$ in this paper correspond to $H, H^{*}, o_{h}, g_{h}, L^{*}, o_{l}, g_{l}$ and $T_{V}$ in [10] respectively; quotient-metrics, quotient-orientations and quotient-forms in this paper correspond to level-metrics, level-orientations and level-forms in [10] respectively. We change notations of [10] because it seems more appropriate to use $L$ to denote a tangent line field and $Q^{*}$ to denote the dual cotangent plane field of $L$, since $Q^{*}$ can be canonically identified as the dual of the intrinsic quotient bundle $T M / L$ of $L$ as mentioned in the beginning of this section.

Now from (2.1) we have

$$
\begin{aligned}
& \bigwedge^{j} T^{*} M=L^{*} \wedge \bigwedge^{j-1} Q^{*} \oplus \bigwedge^{j} Q^{*}, \\
& \Omega^{j}(M)=\overline{L^{*}} \wedge \Omega_{q}^{j-1}(M) \oplus \Omega_{q}^{j}(M), \\
& \Omega^{j}(E)=\overline{L^{*}} \wedge \Omega_{q}^{j-1}(E) \oplus \Omega_{q}^{j}(E),
\end{aligned}
$$

where $\overline{L^{*}}$ denotes the space of all smooth sections of $L^{*}$. Let $\pi_{l}$ be the projection to the first factor and $\pi_{q}$ be the projection to the second factor.

Recall (1.2) that a fiberwise Riemannian metric $g_{q}$ on $Q^{*}$ and an orientation $o_{q}$ on $Q^{*}$ induce the quotient-star operator

$$
*_{q}: \bigwedge^{j} Q^{*} \rightarrow \bigwedge^{2 m-j} Q^{*} .
$$

When $m$ is even we have $*_{q}^{2}=1$ on $\bigwedge^{m} Q^{*}$. When $m$ is odd we have $*_{q}^{2}=-1$ on $\bigwedge^{m} Q^{*}$. In such a case, we let $Q_{c}^{*}=\mathbb{C} \otimes_{\mathbb{R}} Q^{*}$ and identify $Q^{*}$ as $\mathbb{R} \otimes_{\mathbb{R}} Q^{*}$ by the map 
which sends $\alpha$ to $1 \otimes \alpha$. Also we shall write $c \otimes \alpha$ briefly as $c \alpha$ for $\alpha \in Q^{*}$ and $c \in \mathbb{C}$. $Q_{c}^{*}$ has an obvious complex structure $J$ defined as $J(\alpha)=i \alpha$ and $J(i \alpha)=-\alpha$ for $\alpha \in Q^{*}$. We can extend $*_{q}$ complex-linearly to $\bigwedge^{j} Q_{c}^{*} \stackrel{\text { def }}{=} \mathbb{C} \otimes_{\mathbb{R}} \bigwedge^{j} Q^{*}$ for all $j$. The Hermitian quotient-metric $g_{q}^{c}$ on $Q_{c}^{*}$ is defined as

$$
\alpha \wedge *_{q} \bar{\beta}=g_{q}^{c}(\alpha, \beta) \operatorname{vol}\left(g_{q}\right),
$$

where $\bar{\beta}$ is the complex conjugate of $\beta$ with respect to the complex structure $J$. Since $*_{q}$ extends complex-linearly, we still have $*_{q}^{2}=-1$ on $\bigwedge^{m} Q_{c}^{*}$.

Let $\bigwedge_{ \pm}^{m} Q^{*}$ be the \pm 1 -eigenbundle of $*_{q}$ for even $m$, and let $\bigwedge_{ \pm}^{m} Q_{c}^{*}$ be the $\pm i$ eigenbundle of $*_{q}$ for odd $m$.

Let $\Omega_{ \pm}^{m}(M)$ be the space of sections of $\bigwedge_{ \pm}^{m} Q^{*}$ when $m$ is even, and be the space of sections $\bigwedge_{ \pm}^{m} Q_{c}^{*}$ when $m$ is odd.

Let $\Omega_{ \pm}^{m}(E)$ be the space of sections of $\bigwedge_{ \pm}^{m} Q^{*} \otimes_{\mathbb{R}} E$ when $m$ is even, and be the space of sections $\bigwedge_{ \pm}^{m} Q_{c}^{*} \otimes_{\mathbb{C}} E$ when $m$ is odd.

Then we have the following orthogonal decompositions

$$
\begin{aligned}
& \bigwedge^{m} Q^{*}=\bigwedge_{+}^{m} Q^{*} \oplus \bigwedge_{-}^{m} Q^{*} \quad \text { if } m \text { is even, } \\
& \bigwedge^{m} Q_{c}^{*}=\bigwedge_{+}^{m} Q_{c}^{*} \oplus \bigwedge_{-}^{m} Q_{c}^{*} \quad \text { if } m \text { is odd } \\
& \Omega_{q}^{m}(M)=\Omega_{+}^{m}(M) \oplus \Omega_{-}^{m}(M) \\
& \Omega_{q}^{m}(E)=\Omega_{+}^{m}(E) \oplus \Omega_{-}^{m}(E) .
\end{aligned}
$$

Here note that $\Omega_{q}^{m}(M)$ and $\Omega_{q}^{m}(E)$ have been defined in Convention 2.2. The sections of $\bigwedge_{+}^{m} Q^{*}$ and $\bigwedge_{+}^{m} Q_{c}^{*}$ are called self-dual quotient $m$-forms, and those of $\bigwedge_{-}^{m} Q^{*}$ and $\bigwedge_{-}^{m} Q_{c}^{*}$ called anti-self-dual quotient $m$-forms. Let $\pi_{q}^{+}$and $\pi_{q}^{-}$denote the projection to the first and second factor in (2.3) respectively. Define

$$
\begin{aligned}
& \Omega_{++}^{m}(E) \stackrel{\text { def }}{=} \overline{L^{*}} \wedge \Omega_{q}^{m-1}(E) \oplus \Omega_{+}^{m}(E), \\
& \Omega_{+}^{m+1}(E) \stackrel{\text { def }}{=} \overline{L^{*}} \wedge \Omega_{+}^{m}(E) .
\end{aligned}
$$

Let $\pi^{++}$be the projection from $\Omega^{m}(E)$ to $\Omega_{++}^{m}(E)$. Let $\pi^{+}$be the projection from $\Omega^{m+1}(E)$ to $\Omega_{+}^{m+1}(E)$. (Note the notational difference between the terms $\pi^{+}$and $\pi_{q}^{+}$.) Define

$$
d_{A}^{++} \stackrel{\text { def }}{=} \pi^{++} d_{A}, \quad d_{A}^{+} \stackrel{\text { def }}{=} \pi^{+} d_{A} .
$$

Now we want to introduce a parameter $s$ into these operators. The reason to introduce the parameter $s$ is as follows. Computations show that when $|s|^{2}=2$ the symbol of the self-duality operator in Definition 2.3 defines a Clifford module structure. (See Proposition 6.6 and Lemma 6.7.) But in relevant papers [11] and [12] where we need to quote results from this paper, we find it is more natural to work with $s=1$. So we would like to work with a general nonzero parameter $s$ in this paper and define

$$
d_{A}^{++s} \stackrel{\text { def }}{=} l_{s} d_{A}^{++}, \quad d_{A}^{+s} \stackrel{\text { def }}{=} d_{A}^{+} h_{s},
$$


where $l_{s}$ and $h_{s}$ are automorphisms of $\Omega_{++}^{m}(E)$ given by $l_{s}(\theta+\alpha)=\theta+s \alpha$ and $h_{s}(\theta+\alpha)=s \theta+\alpha$, where $\theta \in \overline{L^{*}} \wedge \Omega_{q}^{m-1}(E), \alpha \in \Omega_{+}^{m}(M)$, and $s$ is a nonzero real or complex number. Obviously we have $l_{s} h_{s}=h_{s} l_{s}=s$ on $\Omega_{++}^{m}(E)$.

Given a Riemannian or Hermitian fiberwise metric on $E$, we can define formal adjoints $\left(d_{A}^{++s}\right)^{*}$ and $\left(d_{A}^{+s}\right)^{*}$ in the usual fashion. It is easy to see

$$
\left(d_{A}^{++s}\right)^{*}=d_{A}^{*} l_{\bar{s}}, \quad\left(d_{A}^{+s}\right)^{*}=h_{\bar{s}} \pi^{++} d_{A}^{*},
$$

where $\bar{s}$ is the conjugate of $s$.

Now we are ready to construct the self-duality operator. Let

$$
\begin{gathered}
\Omega_{+}^{\text {od }}(E) \stackrel{\text { def }}{=} \sum_{j=0}^{\frac{m-3}{2}} \Omega^{2 j+1}(E) \oplus \Omega_{++}^{m}(E) \quad \text { for odd } m ; \\
\Omega_{+}^{e v}(E) \stackrel{\text { def }}{=} \sum_{j=0}^{\frac{m-1}{2}} \Omega^{2 j}(E) \oplus \Omega_{+}^{m+1}(E) \quad \text { for odd } m ; \\
\Omega_{+}^{\text {od }}(E) \stackrel{\text { def }}{=} \sum_{j=0}^{\frac{m-2}{2}} \Omega^{2 j+1}(E) \oplus \Omega_{+}^{m+1}(E) \quad \text { for even } m ; \\
\Omega_{+}^{\text {ev }}(E) \stackrel{\text { def }}{=} \sum_{j=0}^{\frac{m-2}{2}} \Omega^{2 j}(E) \oplus \Omega_{++}^{m}(E) \quad \text { for even } m .
\end{gathered}
$$

Let $\omega_{j}$ denote forms of degree $j$. All forms of negative degree are defined to be zero.

Definition 2.3. Let $M$ be a smooth orientable $(2 m+1)$-manifold. The self-duality operator $T_{A}$, associated to data $\left(L, g, o_{q}\right)$ in Definition 2.1 and a connection $A$ on a vector bundle $E$, is defined as

$$
\begin{gathered}
T_{A}: \Omega_{+}^{o d}(E) \rightarrow \Omega_{+}^{e v}(E), \\
T_{A}\left(\sum_{j=0}^{\frac{m-3}{2}} \omega_{2 j+1}+\omega_{m}\right)=\sum_{j=0}^{\frac{m-3}{2}}\left(d_{A} \omega_{2 j-1}+d_{A}^{*} \omega_{2 j+1}\right) \\
+d_{A} \omega_{m-2}+\left(d_{A}^{++s}\right)^{*} \omega_{m}+d_{A}^{+s} \omega_{m} \text { for odd } m ; \\
T_{A}\left(\sum_{j=0}^{\frac{m-2}{2}} \omega_{2 j+1}+\omega_{m+1}\right)= \\
\sum_{j=0}^{\frac{m-2}{2}}\left(d_{A} \omega_{2 j-1}+d_{A}^{*} \omega_{2 j+1}\right) \\
+d_{A}^{++s} \omega_{m-1}+\left(d_{A}^{+s}\right)^{*} \omega_{m+1} \text { for even } m .
\end{gathered}
$$

It is easy to see its formal adjoint is

$$
\begin{gathered}
T_{A}^{*}: \Omega_{+}^{e v}(E) \rightarrow \Omega_{+}^{o d}(E) \\
T_{A}^{*}\left(\sum_{j=0}^{\frac{m-1}{2}} \omega_{2 j}+\omega_{m+1}\right)=\sum_{j=0}^{\frac{m-3}{2}}\left(d_{A} \omega_{2 j}+d_{A}^{*} \omega_{2 j+2}\right) \\
\quad+d_{A}^{++s} \omega_{m-1}+\left(d_{A}^{+s}\right)^{*} \omega_{m+1} \text { for odd } m
\end{gathered}
$$




$$
\begin{aligned}
T_{A}^{*}\left(\sum_{j=0}^{\frac{m-2}{2}} \omega_{2 j}+\omega_{m}\right)= & \sum_{j=0}^{\frac{m-4}{2}}\left(d_{A} \omega_{2 j}+d_{A}^{*} \omega_{2 j+2}\right) \\
& \quad+d_{A} \omega_{m-2}+\left(d_{A}^{++s}\right)^{*} \omega_{m}+d_{A}^{+s} \omega_{m} \text { for even } m .
\end{aligned}
$$

Define $T=T_{A}$ for $d_{A}=d$, when we replace $E$ by $M$ in the above.

Remark 2.4. Although we use $o_{l}$ to define $T_{A}$ in Definitions 2.3, the $o_{l}$ and $-o_{l}$ give the same $T_{A}$ because $*$ appears twice in $d_{A}^{*}$. Thus the $o_{l}$ is not a piece of data for the definition of $T_{A}$.

For both even $m$ and odd $m$, let

$$
\begin{gathered}
\Omega_{+}^{\text {total }}(E) \stackrel{\text { def }}{=} \bigoplus_{j=0}^{m-1} \Omega^{j}(E) \oplus \Omega_{++}^{m}(E) \oplus \Omega_{+}^{m+1}(E), \\
\bar{T}_{A} \stackrel{\text { def }}{=} T_{A} \oplus T_{A}^{*}: \Omega_{+}^{\text {total }}(E) \rightarrow \Omega_{+}^{\text {total }}(E) .
\end{gathered}
$$

Explicitly,

$$
\begin{aligned}
& \bar{T}_{A}\left(\sum_{j=0}^{m-1} \omega_{j}+\omega_{m}+\omega_{m+1}\right) \\
&=\sum_{j=0}^{m-2}\left(d_{A} \omega_{j-1}+d_{A}^{*} \omega_{j+1}\right)+d_{A} \omega_{m-2}+\left(d_{A}^{++s}\right)^{*} \omega_{m} \\
& \quad+d_{A}^{++s} \omega_{m-1}+\left(d_{A}^{+s}\right)^{*} \omega_{m+1}+d_{A}^{+s} \omega_{m} \\
&= \sum_{j=0}^{m-2}\left(d_{A} \omega_{j-1}+d_{A}^{*} \omega_{j+1}\right)+d_{A} \omega_{m-2}+d_{A}^{*} l_{\bar{s}} \omega_{m} \\
& \quad+l_{s} \pi^{++} d_{A} \omega_{m-1}+h_{\bar{s}} \pi^{++} d_{A}^{*} \omega_{m+1}+\pi^{+} d_{A} h_{s} \omega_{m} .
\end{aligned}
$$

A Riemannian metric $g$ is called $L$-admissible if $L \perp_{g} \partial M$ with respect to $g$.

Lemma 2.5. If a tangent line field $L$ on a smooth manifold $M$ is smooth and transverse to $\partial M$ at any point of $\partial M$, then $L$-admissible smooth Riemannian metrics always exist on $M$.

Indeed, we can choose a smooth Riemannian metric $g_{\partial M}$ on the boundary $\partial M$ and a smooth fiberwise Riemannian metric $g_{l}$ on $L$ over $M$. Then for any extension metric of $\left.g_{\partial M} \oplus g_{l}\right|_{\partial M}$ over $M$ we have $L \perp_{g} \partial M$.

Now let $K$ be the union of some connected components of $\partial M$. (It may be empty.) For any data $\left(L, g, o_{q}\right)$ such that $L \perp_{g} \partial M$, define the boundary operator $b$ as follows:

$$
b=\pi_{q} \quad \text { on } K, \quad b=\pi_{l} \quad \text { on } \partial M-K .
$$

The boundary condition on forms $\omega \in \Omega_{+}^{\text {total }}(E)$ is

$$
b \omega=0 \quad \text { on } \partial M .
$$

By using Green's formula, one can easily prove that the boundary operator $b$ on $\Omega_{+}^{\text {total }}(E)$ is self-adjoint, in the sense that $\left(T_{A} \omega, \eta\right)=\left(\omega, T_{A}^{*} \eta\right)$ for any $\omega \in \Omega_{+}^{\text {od }}(E)$ and $\eta \in \Omega_{+}^{e v}(E)$ such that $b \omega=0$ on $\partial M$ and $b \eta=0$ on $\partial M$. We shall review 
the definition of $\mathbb{C}-\mathbb{R}_{+}-\mathbb{R}_{-}$ellipticity in the final section. (Here $\mathbb{C}$ is the field of complex numbers. $\mathbb{R}_{+}$and $\mathbb{R}_{-}$are the set of positive and negative real numbers respectively.)

Theorem 2.6. Let $T_{A}$ be the self-duality operator in Definition 2.3 and let $b$ be the boundary operator in (2.14). Then $\left(T_{A} \oplus T_{A}^{*}, b\right)$ is $\mathbb{C}-\mathbb{R}_{+}-\mathbb{R}_{-}$-elliptic and $\left(T_{A} \oplus T_{A}^{*}\right)_{b}$ is self-adjoint for any nonzero number $s$.

Theorem 2.6 will be proved in the final section. Now define

$$
\begin{array}{lc}
H_{+}^{\text {od }}(A, K) \stackrel{\text { def }}{=} \operatorname{Ker}\left(T_{A}\right)_{b}, & H_{+}^{\text {ev }}(A, K) \stackrel{\text { def }}{=} \operatorname{Ker}\left(T_{A}^{*}\right)_{b} \cong \operatorname{Coker}_{A}, \\
b_{+}^{\text {od }}(A, K) \stackrel{\text { def }}{=} \operatorname{dim} H_{+}^{\text {od }}(A, K), & b_{+}^{\text {ev }}(A, K) \stackrel{\text { def }}{=} \operatorname{dim} H_{+}^{e v}(A, K) .
\end{array}
$$

Here recall that dim refers to complex dimensions when $m$ is odd and to real dimensions when $m$ is even. Let

$$
\operatorname{Ind}\left(T_{A}\right)_{b} \stackrel{\text { def }}{=} b_{+}^{o d}(A, K)-b_{+}^{e v}(A, K)
$$

for a data $\left(L, g_{q}, o_{q}\right)$ as in Definition 2.1 such that $H \perp_{g} \partial M$ and $(E, A)$.

Convention 2.7. In this paper when $d_{A}=d$ we replace the term $A$ by $M,\left.A\right|_{K}$ by $K$, and $T_{A}$ by $T$ in all suitable notations. When $K$ is empty we omit mention of the term $K$. When the quotient orientation $o_{q}$ changes to its opposite $-o_{q}$, we change + signs to - signs in all the corresponding notations.

The most basic formula we obtain in this paper is the index formula for $\operatorname{Ind}\left(T_{A}\right)_{b}$. It has many applications we shall discuss in the subsequent sections. For the statement of this index formula we need to review some notations on even-dimensional manifolds. Let $N$ be a connected, oriented closed Riemannian $2 m$-manifold with orientation $o_{N}$ and let $A_{1}$ be a smooth connection on a real or complex vector bundle $E_{1}$ over $N$. When $m$ is odd, a complex $m$-form $\omega$ over $N$ is called self-dual if ${ }^{*}{ }_{N} \omega=i \omega$. When $m$ is even, a real $m$-form $\omega$ over $N$ is called self-dual if $*_{N} \omega=\omega$. Let $\Omega_{+}^{m}\left(N, E_{1}\right)$ be the space of $E_{1}$-valued complex (real resp.) self-dual $m$-forms when $m$ is odd (even resp.). Let $\Omega^{j}\left(N, E_{1}\right)$ be the space of $E_{1}$-valued complex (real resp.) $j$-forms when $m$ is odd (even resp.). Define

$$
\begin{aligned}
& b^{j}\left(A_{1}\right) \stackrel{\text { def }}{=} \operatorname{dim}\left(\operatorname{Ker} d_{A_{1}} \cap \operatorname{Ker} d_{A_{1}}^{*}\right) \text { in } \Omega^{j}\left(N, E_{1}\right) \text { for } 0 \leq j \leq m-2, \\
& b_{+}^{m-1}\left(A_{1}\right) \stackrel{\text { def }}{=} \operatorname{dim}\left(\operatorname{Ker} \pi_{q}^{+} d_{A_{1}} \cap \operatorname{Ker} d_{A_{1}}^{*}\right) \text { in } \Omega^{m-1}\left(N, E_{1}\right), \\
& b_{+}^{m}\left(A_{1}\right) \stackrel{\text { def }}{=} \operatorname{dimKerd} d_{A_{1}}^{*} \text { in } \Omega_{+}^{m}\left(N, E_{1}\right) .
\end{aligned}
$$

Convention 2.8. When $d_{A_{1}}=d$, we replace the term $A_{1}$ by $N$. When the orientation of $N$ changes to the opposite, we change + signs to - signs.

Next we need to make clear about the difference between the $o_{q}$-orientation and the outward orientation $o_{o u t}$ on $\partial M$. The $o_{q}$-orientation of $\partial M$ is induced by the orientation $o_{q}$ on $L$ and $T_{*}(\partial M)=\left.L\right|_{\partial M}$. It is well-defined as long as the quotientorientation $o_{q}$ on $L$ is given, even if $M$ is not oriented. The outward orientation $o_{\text {out }}$ of $\partial M$ is defined by requiring that at points of $\partial M$ the outward normal vector $e_{\text {out }} \wedge o_{\text {out }}=o_{M}$, the orientation of $M$. 
Let $K$ be the union of some components of $\partial M$ and $A$ be a connection over $M$. The restriction of $A$ to $K$ is denoted as $\left.A\right|_{K}$. Define

$$
\begin{aligned}
\chi_{o_{q}}^{+}\left(\left.A\right|_{K}\right) & =\sum_{j=0}^{m-1}(-1)^{j} b^{j}\left(\left.A\right|_{K}\right)+(-1)^{m} b_{+}^{m}\left(\left.A\right|_{K}\right), \\
\chi_{o_{q}}^{-}\left(\left.A\right|_{K}\right) & =\sum_{j=0}^{m-1}(-1)^{j} b^{j}\left(\left.A\right|_{K}\right)+(-1)^{m} b_{-}^{m}\left(\left.A\right|_{K}\right), \\
\chi_{o_{q}}^{+}(K) & =\sum_{j=0}^{m-1}(-1)^{j} b^{j}(K)+(-1)^{m} b_{+}^{m}(K), \\
\chi_{o_{q}}^{-}(K) & =\sum_{j=0}^{m-1}(-1)^{j} b^{j}(K)+(-1)^{m} b_{-}^{m}(K),
\end{aligned}
$$

where + and - are with respect to the $o_{q}$-orientation on $K$. They make sense even if $M$ is not oriented. Note that

$$
\chi_{o_{q}}^{+}(K)=\frac{1}{2}\left(\chi(K)+\sigma_{o_{q}}(K)\right), \quad \chi_{o_{q}}^{-}(K)=\frac{1}{2}\left(\chi(K)-\sigma_{o_{q}}(K)\right),
$$

where $\chi$ denotes the Euler characteristic and $\sigma_{o_{q}}(K)$ denotes the signature of $K$ with the $o_{q}$-orientation. If $m$ is even, then $\sigma_{o_{q}}(K)=b_{+}^{m}(K)-b_{-}^{m}(K)$, where $b_{+}^{m}(K)$ ( $b_{-}^{m}(K)$ resp.) is the dimension of maximal positive (negative resp.) subspace of the intersection form on $H^{m}(K)$ where $K$ takes the $o_{q}$-orientation. If $m$ is odd, then $\sigma_{o_{q}}(K)=0$ and $b_{ \pm}^{m}(K)=\frac{1}{2} b^{m}(K)$, which is independent of orientations of $K$. Thus

$$
\chi_{o_{q}}^{ \pm}(K)=\frac{1}{2} \chi(K) \quad \text { for odd } m .
$$

Theorem 2.9. Let $M$ be a compact orientable smooth $(2 m+1)$-manifold with nonempty boundary $\partial M$. Let $T_{A}$ be the self-duality operator in Definition 2.3 associated to any given data $\left(L, g, o_{q}\right)$ and any $(E, A)$ and any nonzero number $s$ such that $L \perp_{g} \partial M$. Let $b$ be the boundary operator in (2.14). Then for all $m \geq 1$ we have

$$
\begin{aligned}
\operatorname{Ind}\left(T_{A}\right)_{b} & =\frac{1}{2}\left[\chi_{o_{q}}^{+}\left(\left.A\right|_{K}\right)-\chi_{o_{q}}^{+}\left(\left.A\right|_{\partial M-K}\right)\right], \\
\operatorname{Ind} T_{b} & =\frac{1}{2}\left[\chi_{o_{q}}^{+}(K)-\chi_{o_{q}}^{+}(\partial M-K)\right] .
\end{aligned}
$$

In particular for odd $m \geq 1$ we have

$$
\operatorname{Ind} T_{b}=\frac{1}{4}[\chi(K)-\chi(\partial M-K)] .
$$

Recall that we use $T$ to denote $T_{A}$ when $d_{A}=d$. (2.25) becomes (2.26) when $d_{A}=d$. Theorem 2.9 will be proved in the final section by using an index theorem ([13], Theorem 1.9.3). Before we are going to discuss the special cases and applications of it, we want to say something more about $\chi_{o_{q}}^{+}\left(\left.A\right|_{K}\right)$. When $M$ is oriented, 
we let

$$
\begin{aligned}
& \chi^{+}\left(\left.A\right|_{K}\right) \stackrel{\text { def }}{=} \sum_{j=0}^{m-1}(-1)^{j} b^{j}\left(\left.A\right|_{K}\right)+(-1)^{m} b_{+}^{m}\left(\left.A\right|_{K}\right), \\
& \chi^{-}\left(\left.A\right|_{K}\right) \stackrel{\text { def }}{=} \sum_{j=0}^{m-1}(-1)^{j} b^{j}\left(\left.A\right|_{K}\right)+(-1)^{m} b_{-}^{m}\left(\left.A\right|_{K}\right),
\end{aligned}
$$

where + and - are with respect to the outnormal orientation out on $K$. To illustrate the difference between $\chi_{o_{q}}^{+}\left(\left.A\right|_{K}\right)$ and $\chi^{+}\left(\left.A\right|_{K}\right)$, consider the product case $M=N \times[0,1]$, where $N$ is an oriented closed Riemannian $2 m$-manifold with orientation $o_{N}$ and $[0,1]$ is oriented upward. Since $o_{q}=o_{N}=o_{\text {out }}$ on $N \times\{1\}$ and $o_{q}=o_{N}=-o_{o u t}$ on $N \times\{0\}$, we have $\chi_{o_{q}}^{+}\left(\left.A\right|_{\partial M}\right)=2 \chi^{+}\left(\left.A\right|_{N}\right)$, but $\chi^{+}\left(\left.A\right|_{\partial M}\right)=$ $\chi^{+}\left(\left.A\right|_{N}\right)+\chi^{-}\left(\left.A\right|_{N}\right)$.

Now we discuss the index formulas $(2.25),(2.26)$ and (2.27) in the cases of 3manifolds and 5-manifolds.

Let $M$ be a compact orientable 3-manifold with boundary, and let $A$ be a $U(r)$ connection on a $U(r)$-bundle $E$ over $M$. Let $N$ be a connected component of $\partial M$. The metric $\left.g_{q}\right|_{N}$ and the orientation $o_{q}$ on $\left.Q^{*}\right|_{N}=T^{*} N$ give a complex structure on $N$ by $J=-*_{q}$ on $T^{*} N$. Let $D$ denote $\left.A\right|_{N}$ for a moment. Then $\left.\Lambda_{+}^{1} Q_{c}^{*}\right|_{N}=T^{0,1} N$ and $\pi_{q}^{+} d_{D}=\bar{\partial}_{D}$. Thus the Riemann-Roch Theorem gives

$$
\chi_{o_{q}}^{+}\left(\left.A\right|_{N}\right)=\operatorname{dimKer} \bar{\partial}_{D}-\operatorname{dimCoker} \bar{\partial}_{D}=c_{1}\left(\left.E\right|_{N}\right)+r(1-g(N)) .
$$

For the union $K$ of some components of $\partial M$, let $c_{1}\left(\left.E\right|_{K}\right)$ and $g(K)$ be the sum of $c_{1}\left(\left.E\right|_{N}\right)$ and $g(N)$ over all components $N$ of $K$ respectively.

Corollary 2.10. Let $\left(M, L, g, o_{q}, T_{A}, b, s\right)$ be as in Theorem 2.9 such that $\operatorname{dim} M=$ 3 and $A$ is a $U(r)$-connection on a $U(r)$-bundle $E$ over $M$. Then

$$
\begin{aligned}
\operatorname{Ind}\left(T_{A}\right)_{b}= & \frac{1}{2} c_{1}\left(\left.E\right|_{K}\right)-\frac{1}{2} c_{1}\left(\left.E\right|_{\partial M-K}\right) \\
& +\frac{1}{2} r\left[b^{0}(K)-g(K)-b^{0}(\partial M-K)+g(\partial M-K)\right] .
\end{aligned}
$$

Now suppose $M$ is a compact orientable smooth 5 -manifold with boundary. Let $G$ be a compact semi-simple Lie group, $A$ be a $G$-connection on a principal $G$ bundle $P$ over $M$, and $a d P$ be the adjoint bundle of Lie algebras associated to the $P$. By $(2.19),-\chi_{o_{q}}^{+}\left(\left.A\right|_{N}\right)$ is just the virtual dimension of the moduli space of irreducible anti-self-dual $G$-connections on $\left.P\right|_{N}$, where $N$ is with $o_{q}$-orientation. Thus (Atiyah, Hitchin and Singer [5], Theorem 6.1) gives

$$
-\chi_{o_{q}}^{+}\left(\left.A\right|_{N}\right)=-p_{1}\left(\left.a d P\right|_{N}\right)-\frac{1}{2} \operatorname{dim} G\left[\chi(N)+\sigma_{o_{q}}(N)\right]
$$

For the union $K$ of some components of $\partial M$, let $p_{1}\left(\left.a d P\right|_{K}\right), c_{2}\left(\left.E\right|_{K}\right)$ and $p_{1}\left(\left.E\right|_{K}\right)$ be the sum of $p_{1}\left(\left.a d P\right|_{N}\right), c_{2}\left(\left.E\right|_{N}\right)$ and $p_{1}\left(\left.E\right|_{N}\right)$ over all components $N$ of $K$. We have

Corollary 2.11. Let $\left(M, L, g, o_{q}, T_{A}, b, s\right)$ be as in Theorem 2.9 such that $\operatorname{dim} M=$ 5 and $A$ is a connection on a principal $G$-bundle $P$ over $M$, where $G$ is a compact 
semi-simple group. Then

$$
\begin{aligned}
\operatorname{Ind}\left(T_{A}\right)_{b}= & -\frac{1}{2} p_{1}\left(\left.a d P\right|_{\partial M-K}\right)+\frac{1}{2} p_{1}\left(\left.a d P\right|_{K}\right) \\
& -\frac{1}{4} \operatorname{dim} G\left[\chi(\partial M-K)+\sigma_{o_{q}}(\partial M-K)-\chi(K)-\sigma_{o_{q}}(K)\right] .
\end{aligned}
$$

In particular if $G=S U(2)$ and $E$ is the complex bundle associated to $P$ and the standard representation $\mathbb{C}^{2}$ of $S U(2)$, then

$$
\begin{aligned}
\operatorname{Ind}\left(T_{A}\right)_{b}= & 4 c_{2}\left(\left.E\right|_{\partial M-K}\right)-4 c_{2}\left(\left.E\right|_{K}\right) \\
& -\frac{3}{4}\left[\chi(\partial M-K)+\sigma_{o_{q}}(\partial M-K)-\chi(K)-\sigma_{o_{q}}(K)\right] .
\end{aligned}
$$

If $G=S O(3)$ and $E$ is the real bundle associated to $P$ and the standard representation $\mathbb{R}^{3}$ of $S O(3)$, then

$$
\begin{aligned}
\operatorname{Ind}\left(T_{A}\right)_{b}= & -p_{1}\left(\left.E\right|_{\partial M-K}\right)+p_{1}\left(\left.E\right|_{K}\right) \\
& -\frac{3}{4}\left[\chi(\partial M-K)+\sigma_{o_{q}}(\partial M-K)-\chi(K)-\sigma_{o_{q}}(K)\right] .
\end{aligned}
$$

Corollary 2.12. If $\left(M ; N_{0}, N_{1}\right)$ is a compact connected oriented smooth 5-dimensional rational homology h-cobordism triad such that $\partial M=N_{1} \sqcup N_{0}$ as oriented manifolds and the $L$ points outward on $N_{1}$ and inward on $N_{0}$,

$$
\operatorname{Ind}\left(T_{A}\right)_{b}=-p_{1}\left(\left.a d P\right|_{N_{1}}\right)-\frac{1}{2} \operatorname{dim} G\left[\chi\left(N_{1}\right)+\sigma\left(N_{1}\right)\right] .
$$

Indeed, $o_{q}=o_{\text {out }}$ on $N_{1}$ and $o_{q}=-o_{\text {out }}$ on $N_{0}$. Thus $\sigma_{o_{q}}(\partial M)=\sigma\left(N_{1}\right)-$ $\sigma\left(N_{0}\right)=2 \sigma\left(N_{1}\right)$ and $p_{1}\left(\left.a d P\right|_{\partial M}\right)=2 p_{1}\left(\left.a d P\right|_{N_{1}}\right)$. Note also that since $\left(M ; N_{0}, N_{1}\right)$ is a rational homology $h$-cobordism, we have $\chi(\partial M)=\chi\left(N_{0}\right)+\chi\left(N_{1}\right)=2 \chi\left(N_{1}\right)$.

Remark 2.13. $\operatorname{Ind}\left(T_{A}\right)_{b}$ is the virtual dimension of the moduli space of anti-selfdual $G$-connections on $P$ over $M$ studied in [11]. In the situation as in Corollary 2.12 , it is the same as the dimension of the moduli space of anti-self-dual $G$-connections on $\left.P\right|_{N_{1}}$ over the 4-manifold $N_{1}$, which is the basic object in the Donaldson theory of 4-manifolds. It should be important to study the degree of the restriction mapping from the moduli space of $P$ over $M$ to the moduli space of $\left.P\right|_{N_{1}}$ over $N_{1}$.

\section{An APPLicAtion to NOWHERE ZERO VeCTOR FIELDS}

An immediate consequence of the index formula in Theorem 2.9 is as follows.

Corollary 3.1. Let $M$ be a compact oriented smooth $(2 m+1)$-manifold with nonempty boundary $\partial M$ and $N$ be the union of some components of $\partial M$. If $M$ admits a nowhere zero smooth vector field pointing outward on $N$ and inward on $\partial M-N$, then $\chi(N) \equiv \chi(\partial M-N)(\bmod 4)$.

Proof. Give an orientation on $M$ and let $N$ be the union of the boundary components of $M$ where $L$ points outward. Let $K^{c} \stackrel{\text { def }}{=} \partial M-K$ and $N^{c} \stackrel{\text { def }}{=} \partial M-N$. Then $o_{q}=o_{\text {out }}$ on $N$ and $o_{q}=-o_{\text {out }}$ on $\partial M-N$. Thus we have $\sigma_{o_{q}}(N)=\sigma(N)=$ $\sigma_{o_{q}}\left(N^{c}\right)$. Note that $\sigma(N)+\sigma\left(N^{c}\right)=\sigma(\partial M)=0$. So we have

$$
\sigma\left(N \cap K^{c}\right)+\sigma\left(N^{c} \cap K^{c}\right)+\sigma(N \cap K)+\sigma\left(N^{c} \cap K\right)=0 .
$$

Since

$$
\sigma_{o_{q}}\left(K^{c}\right)=\sigma\left(N \cap K^{c}\right)-\sigma\left(N^{c} \cap K^{c}\right), \quad \sigma_{o_{q}}(K)=\sigma(N \cap K)-\sigma\left(N^{c} \cap K\right),
$$


we have $\sigma_{o_{q}}(K)-\sigma_{o_{q}}\left(K^{c}\right)=2 \sigma(N \cap K)+2 \sigma\left(N^{c} \cap K^{c}\right)$. So by (2.23) we get

$$
\frac{1}{2}\left(\chi_{o_{q}}^{+}(K)-\chi_{o_{q}}^{+}\left(K^{c}\right)\right)=\frac{1}{4}\left[\chi(K)-\chi\left(K^{c}\right)+2 \sigma(N \cap K)+2 \sigma\left(N^{c} \cap K^{c}\right)\right] .
$$

Note that $\chi(K)-\chi\left(K^{c}\right)=2 \chi(K)-\chi(\partial M), \chi(K) \equiv \sigma(K)(\bmod 2)$ and $\chi(N) \equiv$ $\sigma(N)(\bmod 2)$. Therefore when $\bmod 4$, the following identity holds:

$$
\begin{aligned}
0 & \equiv 2 \chi(K)-\chi(\partial M)+2 \sigma(N \cap K)+2 \sigma\left(N^{c} \cap K^{c}\right) \\
& \equiv 2 \sigma(K)-2 \sigma\left(N^{c} \cap K^{c}\right)+2 \sigma(N \cap K)-\chi(\partial M) \\
& \equiv 2 \sigma\left(N \cap K^{c}\right)+2 \sigma(N \cap K)-\chi(\partial M) \\
& =2 \sigma(N)-\chi(\partial M) \equiv \chi(N)-\chi(\partial M-N) .
\end{aligned}
$$

According to Theorem 2.9, the right side of (3.1) must be an integer. Thus we have $\chi(N) \equiv \chi(\partial M-N)(\bmod 4)$.

Corollary 3.1 can be regarded as " $\bmod 4$ " version of the necessity part of Relative Hopf Theorem 1.12 in odd-dimensions, which we shall discuss in Section 3. While the classical proof of Relative Hopf Theorem 1.12 uses essentially homology theory, the proof of Corollary 3.1 in this paper only uses index theory. This is similar to the method in (Atiyah [2]).

\section{The SElF-DuAlity COHOMOlOGY GROUPS OF A GENERAL Line FiELD}

Definition 4.1. Let $0 \leq j \leq m-2$. Define

$\mathcal{H}^{j}(A, K)$ as the space of $\omega \in \operatorname{Ker} d_{A} \cap \operatorname{Ker} d_{A}^{*} \subset \Omega^{j}(E)$ with $b \omega=0$ on $\partial M$,

$\mathcal{H}_{+}^{m-1}(A, K)$ as the space of $\omega \in \operatorname{Ker} d_{A}^{++s} \cap \operatorname{Ker} d_{A}^{*} \subset \Omega^{m-1}(E)$ with $b \omega=0$ on $\partial M$,

$\mathcal{H}_{+s}^{m}(A, K)$ as the space of $\omega \in \operatorname{Ker} d_{A}^{+s} \cap \operatorname{Ker}\left(d_{A}^{++s}\right)^{*} \subset \Omega_{++}^{m}(E)$ with $b \omega=0$ on $\partial M$,

$\mathcal{H}_{+}^{m+1}(A, K)$ as the space of $\omega \in \operatorname{Ker}\left(d_{A}^{+s}\right)^{*} \subset \Omega_{+}^{m+1}(E)$ with $b \omega=0$ on $\partial M$.

They are called the self-duality cohomology groups associated to $\left(L, g, o_{q}, o_{l}\right)$. Let $b^{j}(A, K), \quad b_{+}^{m-1}(A, K), \quad b_{+s}^{m}(A, K) \quad$ and $\quad b_{+}^{m+1}(A, K) \quad$ be $\operatorname{dim}_{\mathcal{H}^{j}}(A, K)$, $\operatorname{dim} \mathcal{H}_{+}^{m-1}(A, K), \operatorname{dim} \mathcal{H}_{+s}^{m}(A, K)$ and $\operatorname{dim} \mathcal{H}_{+}^{m+1}(A, K)$ respectively.

Since $d_{A}^{++s}=l_{s} d_{A}^{++}$and $\left(d_{A}^{+s}\right)^{*}=h_{\bar{s}} \pi^{++} d_{A}^{*}$, we see that $\mathcal{H}_{+}^{m-1}(A, K)$ and $\mathcal{H}_{+}^{m+1}(A, K)$ are independent of $s$.

Condition 4.2. In the case $m=2$, assume $d_{A}^{++} d_{A}=0$ on $\Omega^{0}(E)$. In the case $m \geq 3$, assume $F_{A}=0$. In the case $m=1, A$ can be any connection.

Note that Condition 4.2 clearly holds if $A$ is a flat connection, in particular if $d_{A}=d$.

If Condition 4.2 holds, then by using the Green's formula, we have $d_{A} \omega_{2 j-1} \perp$ $d_{A}^{*} \omega_{2 j+1}, d_{A} \omega_{2 j} \perp d_{A}^{*} \omega_{2 j+2}$ and $d_{A} \omega_{m-2} \perp\left(d_{A}^{++s}\right)^{*} \omega_{m}$ for those $j$ 's in Definition 2.3. Moreover, $d_{A}^{++s} \omega_{m-1}=0$ and $\left(d_{A}^{+s}\right)^{*} \omega_{m+1}=0$ trivially imply that $d_{A}^{++s} \omega_{m-1}+\left(d_{A}^{+s}\right)^{*} \omega_{m+1}=0$. (Note that the converse is not necessarily true in general.) Therefore according to (2.16) and Definitions 2.3 and 4.1, we can have

Proposition 4.3. If $d_{A}=d$ or Condition 4.2 holds, then:

In the case $m$ is odd, we have

$$
H_{+}^{o d}(A, K)=\sum_{j=0}^{\frac{m-3}{2}} \mathcal{H}^{2 j+1}(A, K) \oplus \mathcal{H}_{+s}^{m}(A, K),
$$




$$
\begin{gathered}
H_{+}^{e v}(A, K) \supset \sum_{j=0}^{\frac{m-3}{2}} \mathcal{H}^{2 j}(A, K) \oplus \mathcal{H}_{+}^{m-1}(A, K) \oplus \mathcal{H}_{+}^{m+1}(A, K), \\
b_{+}^{o d}(A, K)=\sum_{j=0}^{\frac{m-3}{2}} b^{2 j+1}(A, K)+b_{+s}^{m}(A, K), \\
b_{+}^{e v}(A, K) \geq \sum_{j=0}^{\frac{m-3}{2}} b^{2 j}(A, K)+b_{+}^{m-1}(A, K)+b_{+}^{m+1}(A, K) .
\end{gathered}
$$

In the case of $m$ is even, we have

$$
H_{+}^{e v}(A, K)=\sum_{j=0}^{\frac{m-2}{2}} \mathcal{H}^{2 j}(A, K) \oplus \mathcal{H}_{+s}^{m}(A, K),
$$

$$
H_{+}^{o d}(A, K) \supset \sum_{j=0}^{\frac{m-4}{2}} \mathcal{H}^{2 j+1}(A, K) \oplus \mathcal{H}_{+}^{m-1}(A, K) \oplus \mathcal{H}_{+}^{m+1}(A, K),
$$

$$
b_{+}^{e v}(A, K)=\sum_{j=0}^{\frac{m-2}{2}} b^{2 j}(A, K) \oplus b_{+s}^{m}(A, K),
$$

$$
b_{+}^{o d}(A, K) \geq \sum_{j=0}^{\frac{m-4}{2}} b^{2 j+1}(A, K) \oplus b_{+}^{m-1}(A, K) \oplus b_{+}^{m+1}(A, K) .
$$

The inequalities in Proposition 4.3 become equalities under the following condition, which is stronger than Condition 4.2.

Condition 4.4. In the case $m=1$, we assume $d_{A}^{+s} d_{A}^{++s}=0$ on $\Omega^{0}(E)$. In the case $m=2$, we assume $d_{A}^{++s} d_{A}=0$ on $\Omega^{0}(E)$ and $d_{A}^{+s} d_{A}^{++s}=0$ on $\Omega^{1}(E)$. In the case $m \geq 3$, we assume $F_{A}=0$ and $d_{A}^{+s} d_{A}^{++s}=0$ on $\Omega^{m-1}(E)$. In addition we assume $L \perp_{g} \partial M$.

If Condition 4.4 holds, we can easily show that $d_{A} \omega_{m-2} \perp\left(d_{A}^{++s}\right)^{*} \omega_{m}$ in Definition 2.3, and thus $d_{A} \omega_{m-2}+\left(d_{A}^{++s}\right)^{*} \omega_{m}=0$ imply $d_{A} \omega_{m-2}=0$ and $\left(d_{A}^{++s}\right)^{*} \omega_{m}=0$. Therefore, we get

Proposition 4.5. If Condition 4.4 holds, then the $\supset$ 's and $\geq$ 's in Proposition 4.3 become equal signs, and for all $m \geq 1$ the following identity holds

$$
\begin{aligned}
\operatorname{Ind}\left(T_{A}\right)_{b}= & \sum_{j=0}^{m-2}(-1)^{j+1} b^{j}(A, K)+(-1)^{m} b_{+}^{m-1}(A, K) \\
& +(-1)^{m+1} b_{+}^{m}(A, K)+(-1)^{m} b_{+}^{m+1}(A, K) .
\end{aligned}
$$

By Theorem 2.6, $\left(T_{A}\right)_{b}$ is a Fredholm operator for all nonzero numbers $s$. Indices of Fredholm operators are deformation invariant. Thus under Condition 4.4, $b_{+s}^{m}(A, K)$ is independent of $s$, and can be denoted as $b_{+}^{m}(A, K)$. 
Theorem 4.6. Let $M$ be a compact connected orientable smooth $(2 m+1)$-manifold with nonempty boundary with data $\left(L, g, o_{q}\right)$ such that $L \perp_{g} \partial M$. Let $K$ be the union of some components of $\partial M$. Then

(1) For any $m \geq 1$, we have

$$
\mathcal{H}_{+}^{m-1}(M, K) \cong H^{m-1}(M, K), \quad b_{+}^{m-1}(M, K)=b^{m-1}(M, K) .
$$

In the case $m$ is odd, we further have

$$
\begin{gathered}
H_{+}^{\text {od }}(M, K)=\sum_{j=0}^{\frac{m-3}{2}} \mathcal{H}^{2 j+1}(M, K) \oplus \mathcal{H}_{+s}^{m}(A, K), \\
H_{+}^{e v}(M, K) \supset \sum_{j=0}^{\frac{m-1}{2}} \mathcal{H}^{2 j}(M, K) \oplus \mathcal{H}_{+}^{m+1}(M, K), \\
b_{+}^{\text {od }}(M, K)=\sum_{j=0}^{\frac{m-3}{2}} b^{2 j+1}(M, K)+b_{+s}^{m}(M, K), \\
b_{+}^{e v}(M, K) \geq \sum_{j=0}^{\frac{m-1}{2}} b^{2 j}(M, K)+b_{+}^{m+1}(M, K), \\
b_{+}^{\text {od }}(M, K)=b_{+}^{e v}(M, K)+\frac{1}{4}[\chi(K)-\chi(\partial M-K)] .
\end{gathered}
$$

In the case $m$ is even, we further have

$$
\begin{gathered}
H_{+}^{e v}(M, K)=\sum_{j=0}^{\frac{m-2}{2}} \mathcal{H}^{2 j}(M, K) \oplus \mathcal{H}_{+s}^{m}(M, K), \\
H_{+}^{\text {od }}(M, K) \supset \sum_{j=0}^{\frac{m-2}{2}} \mathcal{H}^{2 j+1}(M, K) \oplus \mathcal{H}_{+}^{m+1}(M, K), \\
b_{+}^{e v}(M, K)=\sum_{j=0}^{\frac{m-2}{2}} b^{2 j}(M, K)+b_{+s}^{m}(M, K), \\
b_{+}^{o d}(M, K) \geq \sum_{j=0}^{\frac{m-2}{2}} b^{2 j+1}(M, K)+b_{+}^{m+1}(M, K), \\
b_{+}^{e v}(M, K)=b_{+}^{o d}(M, K)-\frac{1}{2}\left[\chi_{o_{q}}^{+}(K)-\chi_{o_{q}}^{+}(\partial M-K)\right] .
\end{gathered}
$$

(2) In the case $K \neq \partial M$, for any $m \geq 1$ we have

$$
\mathcal{H}_{+}^{m+1}(M, K)=0, \quad b^{m+1}(M, K)=0 .
$$


Proof. According to ([10], Corollary 7.7), we have

$$
\mathcal{H}_{+}^{m+1}(M, K)=0 \quad \text { and } \quad b_{+}^{m+1}(M, K)=0
$$

in the case $K \neq \partial M$. According to ([10], Corollary 7.5), we have $\mathcal{H}_{+}^{m-1}(M, K)=$ $\mathcal{H}^{m-1}(M, K) \cong H^{m-1}(M, K)$ and $b_{+}^{m-1}(M, K)=b^{m-1}(M, K)$ for any $K$. Then we can apply $I n d T_{b} \stackrel{\text { def }}{=} b_{+}^{o d}(M, K)-b_{+}^{e v}(M, K)$ and Theorem 2.9 and Proposition 4.3 to complete the proof.

By (4.13), (4.14) and (4.15) in the case of odd $m$ and (4.18), (4.19) and (4.20) in the case of even $m$ and (4.21), we have

Corollary 4.7. Let $\left(M, L, o_{q}, g, K\right)$ be as in Theorem 4.6. If $K \neq \partial M$, then

$$
\begin{gathered}
b_{+s}^{m}(M, K) \geq \chi_{\frac{1}{2}}(M, K)+\frac{1}{4}[\chi(K)-\chi(\partial M-K)] \quad \text { for odd } m, \\
b_{+s}^{m}(M, K) \geq-\chi_{\frac{1}{2}}(M, K)-\frac{1}{2}\left[\chi_{o_{q}}^{+}(K)-\chi_{o_{q}}^{+}(\partial M-K)\right] \quad \text { for even } m .
\end{gathered}
$$

\section{Applications to NeARLY-SELF-DUAl Line Fields II}

First we need to give the definition of a nearly-self-dual line field.

Definition 5.1. A tangent line field $L$ on a smooth $(2 m+1)$-manifold $M$ is called nearly-self-dual if it is smooth and there is a smooth fiberwise Riemannian metric $g_{q}$ on $Q^{*}$ and an orientation on $Q^{*}$ such that $\Gamma_{V} \stackrel{\text { def }}{=} L_{V} *_{q}-*_{q} L_{V}=0$ on $d_{q}^{-} \Omega^{m-1}(M) \cup d_{q}^{+} \Omega^{m-1}(M)$ for any smooth section $V$ of $L$ and for a splitting $T M=L \oplus Q$ with $Q$ tangent to $\partial M$. Such a metric $g_{q}$ on $Q^{*}$ is called $L$-compatible. A vector field is called nearly-self-dual if it is nowhere zero, smooth and the line field generated by the vector field is nearly-self-dual. Here $d_{q}^{ \pm}=\pi_{q}^{ \pm} \pi_{q} d$.

Definition 5.2. A smooth Riemannian metric $g$ on a smooth $(2 m+1)$-manifold $M$ with a nearly-self-dual (self-dual resp.) line field $L$ is called $L$-compatible if (1) $L \perp_{g} \partial M$ at any point of $\partial M$ and (2) the induced metric $\left.g_{q} \stackrel{\text { def }}{=} g\right|_{Q^{*}}$ on $Q^{*}$ over $M$ is $L$-compatible in the sense of Definition 5.1 (Definition 1.1 resp.).

Note that when $\partial M$ is empty the condition (1) is automatically satisfied.

Lemma 5.3. If a tangent line field $L$ on a smooth $(2 m+1)$-manifold $M$ is nearlyself-dual (self-dual resp.) and transverse to $\partial M$ at any point of $\partial M$, then $L$ compatible smooth Riemannian metrics always exist on $M$.

Proof. For nearly-self-dual case it is clear. We only need to prove for self-dual case. According to Lemma 2.5, there is a smooth Riemannian metric $g^{\prime}$ on $M$ such that $L \perp_{g^{\prime}} \partial M$ at any point of $\partial M$. Let $L^{*} \stackrel{\text { def }}{=}\left(Q^{*}\right)^{\perp_{g^{\prime}}}$, the $g^{\prime}$-orthogonal complement of $Q^{*}$ in $T^{*} M$. Then $T^{*} M=L^{*} \oplus Q^{*}$. Define $\left.g \stackrel{\text { def }}{=} g^{\prime}\right|_{L^{*}} \oplus g_{q}$, where $g_{q}$ is a $L$-compatible smooth fiberwise Riemannian metric on $Q^{*}$ in Definition 1.1. This $g$ is $L$-compatible, which is proved as follows. We only need to prove $L \perp_{g} \partial M$. For any vector $Y \in T M$, let $Y_{g}$ ( $Y_{g^{\prime}}$ resp. ) denote the covector dual to $Y$ with respect to the metric $g$ ( $g^{\prime}$ resp. ).

Let $\left.V \in L\right|_{\partial M}$ and $W \in T(\partial M)$ at the same point in $\partial M$. By (1.1) and $V \in L$ we have $0=\left\langle V, Q^{*}\right\rangle=g\left(V_{g}, Q^{*}\right)$. Thus $V_{g} \in\left(Q^{*}\right)^{\perp_{g}}=L^{*}$. 
Since $W \in T(\partial M) \perp_{g^{\prime}} L$, we have $0=g^{\prime}(W, L)=\left\langle W_{g^{\prime}}, L\right\rangle$ and thus $W_{g^{\prime}} \in Q^{*}=$ $\left(L^{*}\right)^{\perp} g^{\prime}$ by (1.1) and definition of $L^{*}$. Thus $0=g^{\prime}\left(W_{g^{\prime}}, L^{*}\right)=\left\langle W, L^{*}\right\rangle=g\left(W_{g}, L^{*}\right)$. So $W_{g} \in\left(L^{*}\right)^{\perp_{g}}$, and therefore $0=g\left(V_{g}, W_{g}\right)=g(V, W)$ since $V_{g} \in L^{*}$, shown in the above.

As usual let $i_{V}$ denote the interior contraction by vector field $V$. Define

$$
\nabla_{A_{V}} \stackrel{\text { def }}{=} i_{V} d_{A}, \quad F_{A_{V}} \stackrel{\text { def }}{=} i_{V} F_{A}, \quad d_{A_{q}}=\pi_{q} d_{A}, \quad d_{A_{q}}^{ \pm}=\pi_{q}^{ \pm} d_{A_{q}} .
$$

When $d_{A}=d$ we omit mention of $A$. Now we quote a formula from [10]. It can also be directly checked.

Proposition 5.4 ([10], Proposition 3.4). Let $m$ be any integer $\geq 1$. Let $A$ be any connection on a real or complex vector bundle $E$. Then

$$
d_{A}^{+s} d_{A}^{++s} \omega=s \tau \wedge\left[\frac{\epsilon}{2} \Gamma_{V} d_{A_{q}}^{-} \omega+\pi_{q}^{+}\left(F_{A_{V}} \wedge \alpha\right)+\pi_{q}^{+}\left(F_{A_{q}} \wedge \beta\right)\right]
$$

for all $\omega \in \Omega^{m-1}\left(\left.E\right|_{U}\right)$, where $V$ is a section of $L$ over $U, \tau$ is a section of $L^{*}$ over $U$ such that $\langle\tau, V\rangle=1, \omega=\tau \wedge \beta+\alpha$ with $\beta \in \Omega_{q}^{m-2}\left(\left.E\right|_{U}\right)$ and $\alpha \in \Omega_{q}^{m-1}\left(\left.E\right|_{U}\right)$, $\epsilon=1$ for even $m$ and $\epsilon=-i$ for odd $m$.

Note that when $m=1, \pi_{q}^{+}\left(F_{A_{q}} \wedge \beta\right)=0$ always holds, for $\beta \in \Omega_{q}^{m-2}\left(\left.E\right|_{U}\right)=0$. When $m=2, \pi_{q}^{+}\left(F_{A_{q}} \wedge \beta\right)=\left(\pi_{q}^{+} F_{A_{q}}\right) \beta$, since $\beta$ is a $\left.E\right|_{U}$-valued function.

Since $F_{A}=0, F_{A_{V}}=0$ and $F_{A_{q}}=0$ for $d_{A}=d$, by Proposition 5.4, we see that the conditions in Definition 5.1 imply Condition 4.4 for $d_{A}=d$, and therefore we get the following theorem (cf. [10], Theorems 1.3 and 2.2):

Theorem 5.5. If a tangent line field $L$ on a smooth $(2 m+1)$-manifold $M$ is nearlyself-dual, then for any $L$-compatible metric $g_{q}$ on $Q^{*}$ and any orientation $o_{q}$ on $Q^{*}$ and any nonzero $s$, the following complex is well-defined

$$
0 \rightarrow \Omega^{0}(M) \stackrel{d}{\rightarrow} \cdots \stackrel{d}{\rightarrow} \Omega^{m-1}(M) \stackrel{d^{++s}}{\rightarrow} \Omega_{++}^{m}(M) \stackrel{d^{+s}}{\rightarrow} \Omega_{+}^{m+1}(M) \rightarrow 0 .
$$

Complex (5.3) and its generalizations are the main subjects first studied in [10].

Theorem 5.6. Let $M$ be a compact connected orientable smooth $(2 m+1)$-manifold with nonempty boundary and with data $\left(L, g, o_{q}\right)$ such that $L$ is nearly-self-dual and transverse to $\partial M$ and $g$ is $L$-compatible. Then $b_{ \pm s}^{m}(M, K)$ are independent of $s$, and thus can be denoted as $b_{ \pm}^{m}(M, K)$. Moreover all $\geq$ 's and $\supset$ 's in Theorem 4.6 and 4.7 become equal. Explicitly for all $m$ :

(1) In the case $K \neq \partial M$, we have

(2) In the case $K=\partial M$, we have

$$
\begin{gathered}
b_{ \pm}^{m-1}(M, K)=b^{m-1}(M, K), \\
b_{ \pm}^{m+1}(M, K)=0, \\
b_{ \pm}^{m}(M, K)=(-1)^{m+1} \chi_{\frac{1}{2}}(M, K)+\frac{(-1)^{m+1}}{2}\left[\chi_{o_{q}}^{ \pm}(K)-\chi_{o_{q}}^{ \pm}(\partial M-K)\right] .
\end{gathered}
$$

$$
\begin{gathered}
b_{ \pm}^{m-1}(M, \partial M)=b^{m-1}(M, \partial M) \\
b_{ \pm}^{m}(M, \partial M)-b_{ \pm}^{m+1}(M, \partial M)=(-1)^{m+1} \chi_{\frac{1}{2}}(M, \partial M)+\frac{(-1)^{m+1}}{2} \chi_{o_{q}}^{ \pm}(\partial M)
\end{gathered}
$$


Proof. Since the conditions in Definition 5.1 imply Condition 4.4 for $d_{A}=d$, we can use the conclusion of Proposition 4.5 for $d_{A}=d$. Also, the conditions in Definition 5.1 imply the conditions of Theorem 4.6 and Corollary 4.7. In addition, ([10], Corollary 7.5) gives that $b_{+}^{m-1}(M, K)=b^{m-1}(M, K)$ for all $K$, including the case $K=\partial M$. Thus all these together imply the above formulas about the dimensions of the self-duality cohomology groups.

Theorem 5.7. Let $(M, V, C, N)$ be the same as in Theorem 1.16. Then we have

$$
b_{ \pm}^{m}(C) \geq(-1)^{m} \chi_{\frac{1}{2}}(M, \partial M)+\frac{(-1)^{m}}{4}[\chi(\partial M) \pm 2 \sigma(N)]+b_{+}^{m}(M, \partial M),
$$

where $b_{+}^{m}(M, \partial M)$ is with respect to some $\langle V\rangle$-compatible smooth Riemannian metric $g$ on $M$.

Proof. Since $L \perp_{g} C$ in the case (1) or $L_{V} *_{q}=*_{q} L_{V}$ on $\wedge{ }^{m} Q^{*}$ in the case (2), we can use ([10], Corollary 7.10) to get

$$
b_{ \pm}^{m+1}(M, \partial M) \leq b_{ \pm}^{m}(C) .
$$

Using notations in Theorem 5.6, we have $\chi_{o_{q}}^{ \pm}(\partial M)=\frac{1}{2}(\chi(\partial M) \pm 2 \sigma(N))$. Then (5.8) and (5.10) yield (5.9).

Remark 5.8. Note that Theorem 1.16 follows directly from Theorem 5.7 and Lemma 5.3 and the fact that $b_{+}^{m}(M, \partial M) \geq 0$.

The Relative Hopf Theorem 1.12 applied to a 3-manifold yields the following result about existence of proper vector fields on a 3 -manifold.

Proposition 5.9. Let $M$ be a compact connected oriented 3-manifold with $k$ many boundary components.

(1) In the case $k=1, M$ admits a proper vector field if and only if $\partial M$ is a torus.

(2) In the case $k=2$, let $N_{0}$ and $N_{1}$ be two boundary components of $M$. Then $M$ admits a proper vector field which is outward on $N_{0}$ and inward on $N_{1}$ if and only if $g\left(N_{0}\right)=g\left(N_{1}\right)$. $M$ admits a proper vector field outward (or inward) on both $N_{0}$ and $N_{1}$ if and only if $g\left(N_{0}\right)+g\left(N_{1}\right)=2$.

(3) In the case $k \geq 3, M$ admits a proper vector field if and only if there is a union $N$ of boundary components of $M$ such that $\chi(N)=\frac{1}{2} \chi(\partial M)$.

When $\operatorname{dim} M=3,(5.8)$ gives

$$
b_{+}^{2}(M, \partial M)=b_{+}^{1}(M, \partial M)-\frac{1}{4} \chi(\partial M) .
$$

This and Proposition 5.9 give a lot of information on $b_{+}^{2}(M, \partial M)$ and $b_{+}^{1}(M, \partial M)$.

Proposition 5.10. Let $\left(M, L, g, o_{q}\right)$ be as in Theorem 5.6 with $\operatorname{dim} M=3$ and let $k$ be the number of boundary components of $M$. In the case $k=2$, we let $N_{1}$ and $N_{0}$ be two boundary components of $M$, arranged so that $g\left(N_{0}\right) \leq g\left(N_{1}\right)$. Then one of the following cases must hold:

(1) $k \neq 2, b_{+}^{2}(M, \partial M)=b_{+}^{1}(M, \partial M)=0$ or 1 .

(2) $k=2, g\left(N_{0}\right)=g\left(N_{1}\right), b_{+}^{2}(M, \partial M)=b_{+}^{1}(M, \partial M)+g\left(N_{1}\right)-1, b_{+}^{1}(M, \partial M)=$ 0 or 1 , and $V$ is outward on one boundary component and inward on the other boundary component.

(3) $k=2, g\left(N_{0}\right)=0, g\left(N_{1}\right)=2, b_{+}^{2}(M, \partial M)=b_{+}^{1}(M, \partial M)=0$, and $V$ is outward (or inward) on $N_{1}$ and $N_{0}$. 
(4) $k=2, g\left(N_{0}\right)=g\left(N_{1}\right)=1, b_{+}^{2}(M, \partial M)=b_{+}^{1}(M, \partial M)=0$ or 1 , and $V$ is outward (or inward) on $N_{1}$ and $N_{0}$.

Proof. Let $N$ be a boundary component of $M$. Since $L \perp_{g} N$ and $V$ is nearly-selfdual, we can use ([10], Corollary 7.10) to get

$$
b_{+}^{2}(M, \partial M) \leq b_{+}^{1}(N) .
$$

Recall that $b_{ \pm}^{1}(N)=g(N)$ for closed oriented 2-manifold $N$.

If $k \neq 2$, then by Theorem 1.15, we have $1=g(N) \geq b_{+}^{2}(M, \partial M)=b_{+}^{1}(M, \partial M)$ $\geq 0$.

Now suppose $k=2$. In the case where $V$ points outward on $N_{1}$ and inward on $N_{0}$, we have $g\left(N_{0}\right)=g\left(N_{1}\right)$ and $\chi(\partial M)=4-4 g\left(N_{1}\right)$. Thus $g\left(N_{1}\right) \geq b_{+}^{2}(M, \partial M)=$ $b_{+}^{1}(M, \partial M)-\frac{1}{4}\left(4-4 g\left(N_{1}\right)\right) \geq g\left(N_{1}\right)-1$. This gives the claim (2).

In the case where $V$ points outward (inward) on $N_{1}$ and $N_{0}$, we have $g\left(N_{0}\right)+$ $g\left(N_{1}\right)=2$ and $\chi(\partial M)=0$. Thus $g\left(N_{0}\right) \geq b_{+}^{2}(M, \partial M)=b_{+}^{1}(M, \partial M)$. Since $g\left(N_{0}\right)+g\left(N_{1}\right)=2$ and $g\left(N_{0}\right) \leq g\left(N_{1}\right)$, we have $g\left(N_{0}\right)=0$ or 1 , which yields the claims (3) and (4).

\section{Proofs of Theorems 1.9, 1.15, 2.9 And 2.6}

In this section we shall present in order all the proofs of Theorems 1.9, 1.15, 2.9 and 2.6.

I. Proof of Theorem 1.9 and Theorem 1.15.

By applying Lemma 5.3 and (5.6) and (3.1), we have

$$
\begin{aligned}
0 \leq & b_{+}^{m}(M, K)=(-1)^{m+1} \chi_{\frac{1}{2}}(M, K) \\
& +(-1)^{m+1} \frac{1}{4}\left[\chi(K)-\chi\left(K^{c}\right)+2 \sigma(N \cap K)+2 \sigma\left(N^{c} \cap K^{c}\right)\right], \\
0 \leq & b_{-}^{m}(M, K)=(-1)^{m+1} \chi_{\frac{1}{2}}(M, K) \\
& +(-1)^{m+1} \frac{1}{4}\left[\chi(K)-\chi\left(K^{c}\right)-2 \sigma(N \cap K)-2 \sigma\left(N^{c} \cap K^{c}\right)\right] .
\end{aligned}
$$

Since $\chi(K)-\chi\left(K^{c}\right)=-\chi(\partial M)+2 \chi(K)$, we get (1.10) and thus complete the proof of Theorem 1.9.

Now we are going to prove Theorem 1.15. Suppose $M$ admitted a nearly-self-dual proper vector field. When $k=1, \partial M$ had to be a torus according to Proposition 5.9. This contradicts to the assumption. Suppose $k \geq 3$. Recall (5.6). Since $m=1$, we have $\chi_{\frac{1}{2}}(M, K)=0, \chi_{\frac{1}{2}}\left(M, K^{c}\right)=0$ and $\sigma$ of any boundary component of $M$ is zero. THus (5.6) yields $0 \leq b_{+}^{1}(M, K)=\chi(K)-\chi\left(K^{c}\right)$. Switching $K$ and $K^{c}$, we get that $\chi(K)=\chi\left(K^{c}\right)$ holds for any nonempty union $K$ of components of $\partial M$ such that $K \neq \partial M$. Let $N_{0}, N_{1}, N_{2}, \ldots, N_{k-1}$ be connected components of $\partial M$, $k \geq 3$, and be arranged in order: $\chi_{0} \leq \chi_{1} \leq \ldots \leq \chi_{k-1} \leq 2$, where $\chi_{j}=\chi\left(N_{j}\right)$.

Since $\chi_{0}=\sum_{j=1}^{k-1} \chi_{j}$ and $\chi_{k-1}=\sum_{j=0}^{k-2} \chi_{j}$, we get $\chi_{0}=\chi_{k-1}$. Thus $\chi_{0}=\chi_{j}$ for all $j=1, \ldots, k-1$. Therefore $\chi_{0}=(k-2) \chi_{0}$. Since $k \neq 2$, we got $0=\chi_{0}=\chi_{j}$ for all $j$. This contradicts to the assumption.

II. Proof of Theorem 2.9.

First let us recall an index theorem from [13]. Let $F_{1}$ and $F_{2}$ be complex vector bundles over $M$ with $\operatorname{dim} F_{1}=d i m F_{2}$. Let $F_{1}^{\prime}$ and $F_{2}^{\prime}$ be complex vector bundles over $\partial M$ with $2 \operatorname{dim} F_{i}^{\prime}=\operatorname{dim} F_{i}, i=1,2$. Let $P: \Gamma\left(F_{1}\right) \rightarrow \Gamma\left(F_{2}\right)$ be an elliptic first 
order differential operator with a zero-th order boundary operator $B: \Gamma\left(\left.F_{1}\right|_{\partial M}\right) \rightarrow$ $\Gamma\left(F_{1}^{\prime}\right)$. The operator

$$
(P, B): \operatorname{Dom}(P) \rightarrow \operatorname{Rang}(P) \oplus \operatorname{Rang}(B)
$$

is given by $(P, B) f=\left(P f, B\left(\left.f\right|_{\partial M}\right)\right)$. The operator

$$
P_{B}:\left\{f \in \operatorname{Dom}(P), B\left(\left.f\right|_{\partial M}\right)=0\right\} \rightarrow \operatorname{Rang}(P)
$$

is defined by $P_{B} f=P f$. If $B$ is surjective then $\operatorname{Ind} P_{B}=\operatorname{Ind}(P, B)$. Let $P^{*}$ be the formal adjoint of $P$. The adjoint boundary operator $B^{*}: \Gamma\left(\left.F_{2}\right|_{\partial M}\right) \rightarrow$ $\Gamma\left(F_{2}^{\prime}\right)$ is defined by the requirement that $\left(P f, f^{\prime}\right)=\left(f, P^{*} f^{\prime}\right)$ when $B\left(\left.f\right|_{\partial M}\right)=0$ and $B^{*}\left(\left.f^{\prime}\right|_{\partial M}\right)=0$. Note $P \oplus P^{*}: \Gamma\left(F_{1} \oplus F_{2}\right) \rightarrow \Gamma\left(F_{1} \oplus F_{2}\right)$ and $B \oplus B^{*}$ : $\Gamma\left(\left.F_{1} \oplus F_{2}\right|_{\partial M}\right) \rightarrow \Gamma\left(F_{1}^{\prime} \oplus F_{2}^{\prime}\right)$. The definitions of $\mathbb{C}-\mathbb{R}_{+}-\mathbb{R}_{-}$ellipticity will be reviewed later.

Theorem 6.1 ([13], Theorem 1.9.3). If $\left(P \oplus P^{*}, B \oplus B^{*}\right)$ is $\mathbb{C}-\mathbb{R}_{+}-\mathbb{R}_{-}$-elliptic and $\left(P \oplus P^{*}\right)_{B \oplus B^{*}}$ is self-adjoint, then

$$
\operatorname{IndP} P_{B}=\int_{M} a_{n}(P) \operatorname{vol}(M)+\int_{\partial M} a_{n-1}(P, B) \operatorname{vol}(\partial M) .
$$

Moreover, (1) $a_{n}(P)=0$ if $n=\operatorname{dim} M$ is odd, and (2) $a_{n-1}(P, B)$ is a scalar valued local invariant defined on $Y$ and depends functorially on a finite number of jets of symbols of $P$ and $B$ on the boundary $\partial M$.

Note that $a_{n}(P)$ is the interior local invariant for the index of an elliptic operator $P$ on the double of $M$, and thus is zero if $n$ is odd.

For the boundary operator (2.14), let $K=K_{1} \sqcup \cdots \sqcup K_{l}$ and $\partial M-K=N_{1} \sqcup$ $\cdots \sqcup N_{l}$, where all $N_{j}$ 's and $K_{j}$ 's are components of $\partial M$. Since $\operatorname{dim} M=2 m+1$ is odd, Theorem 6.1 gives

$$
\begin{aligned}
\operatorname{Ind}\left(T_{A}\right)_{b} & =\int_{\partial M} a_{2 m}\left(T_{A}, b\right) \operatorname{vol}(\partial M) \\
& =\sum_{j=1}^{h} \int_{N_{j}} a_{2 m}\left(T_{A}, \pi_{l}\right) \operatorname{vol}\left(N_{j}\right)+\sum_{k=1}^{l} \int_{K_{j}} a_{2 m}\left(T_{A}, \pi_{q}\right) \operatorname{vol}\left(K_{j}\right) .
\end{aligned}
$$

Thus we only need to determine

$$
\int_{N_{j}} a_{2 m}\left(T_{A}, \pi_{l}\right) \operatorname{vol}\left(N_{j}\right) \text { and } \int_{K_{j}} a_{2 m}\left(T_{A}, \pi_{q}\right) \operatorname{vol}\left(K_{j}\right) .
$$

The key property of $a_{2 m}\left(T_{A}, b\right)$ is that it is scalar valued local invariant depends functorially on a finite number of jets of symbols of $T_{A}$ and $b$ on the boundary of $M$. The operators $T_{A}$ and $b$ in Definition 2.3 and (2.14) depend only on the data $\left(L, g, o_{q}, A, E\right)$ and don't depend on the orientation $o_{l}$ on $L$. (See Remark 2.4.) Thus we have

Proposition 6.2. Given two sets of data $\left(L, g, o_{q}, A, E\right)$ and $\left(L^{\prime}, g^{\prime}, o_{q}^{\prime}, A^{\prime}, E^{\prime}\right)$, where $g$ is L-admissible and $g^{\prime}$ is $L^{\prime}$-admissible. Suppose $C$ and $C^{\prime}$ are boundary components of compact oriented $(2 m+1)$-manifolds $M$ and $M^{\prime}$ respectively. If there is an isometry $\phi$ from a collar of $C$ in $M$ onto a collar of $C^{\prime}$ in $M^{\prime}$ such that $\phi$ maps the data $\left(L, g, o_{q}, A, E\right)$ to the data $\left(L_{*}^{\prime}, g^{\prime}, o_{q}^{\prime}, A^{\prime}, E^{\prime}\right)$, then $a_{2 m}\left(T_{A}, b\right)$ at point $y$ of $C$ is the same as $a_{2 m}\left(T_{A}^{\prime}, b^{\prime}\right)$ at point $\phi(y)$ of $C^{\prime}$ and

$$
\int_{C} a_{2 m}\left(T_{A}, b\right) \operatorname{vol}(C)=\int_{C^{\prime}} a_{2 m}\left(T_{A}^{\prime}, b^{\prime}\right) \operatorname{vol}\left(C^{\prime}\right) .
$$


We should emphasize that the above $\phi$ is permissible to map $o_{l}$ to either $o_{l}^{\prime}$ or $-o_{l}^{\prime}$.

Now we compute the index in the simplest case. Let $N$ be a connected, oriented closed $2 m$-manifold with orientation $o_{N}$. Let $M=N \times[0,1]$ with the obvious line field $L$, i.e., the field of tangent lines of copies of $[0,1]$ in $M . Q^{*} \stackrel{\text { def }}{=} L^{\perp}$ is the pull-back of $T^{*} N$ by the projection $\pi_{N}: M \rightarrow N$. The orientation on $N$ gives an orientation $o_{q}$ on $Q^{*}$.

Let $M$ take the orientation $o_{M}=d t \wedge o_{N}$, where $t \in[0,1]$. Then $\partial M=N \times\{1\} \sqcup$ $N \times\{0\}$ gets the outward orientation $o_{\text {out }}$ by $e_{\text {out }} \wedge o_{\text {out }}=o_{M}=d t \wedge o_{N}$, where $e_{\text {out }}$ is a covector field on $\partial M$ pointing out of $M$. On $N \times\{1\}$ we have $o_{q}=o_{\text {out }}$. On $N \times\{0\}$ we have $o_{q}=-o_{\text {out }}$. We can identify $N \times\{1\}$ with $N$ by $(x, 1) \rightarrow x$ and identify $N \times\{0\}$ with $N^{-}$by $(x, 0) \rightarrow x$, where $N^{-}$is the oppositely oriented $N$.

Let $g_{0}$ be the product of a metric on $N$ and the standard metric on [0,1], and let $A_{1}$ be a smooth connection on a real or complex vector bundle $E_{1}$ over $N$. The pull back of $A_{1}$ to the bundle $E=\pi_{N}^{*} E_{1}$ over $M$ is called the pulled-back connection $A$ of $A_{1}$ over $N . d_{A}=d_{A_{1}}+\frac{\partial}{\partial t} d t$. For such an $A$, we have

$$
\pi_{l} F_{A}=0, \quad F_{A}=\pi_{q} F_{A}=F_{A_{1}} .
$$

Let $*_{N}$ be the star operator on $N$. Recall that when $m=2, A_{1}$ is called an anti-self-dual connection if $*_{N} F_{A_{1}}=-F_{A_{1}}$.

Lemma 6.3. Suppose $A_{1}$ is a flat smooth connection over $N$ when $m \geq 3 ; A_{1}$ is an anti-self-dual smooth connection over $N$ when $m=2$; and $A_{1}$ is any given smooth connection when $m=1$. Then the pulled-back connection $A$ of $A_{1}$ satisfies Condition 4.4.

Proof. Since $M=N \times[0,1]$, we can let $\tau=d t$ and $V=\frac{\partial}{\partial t}$ with $t \in[0,1]$. Since $g$ is a product metric, we have $\Gamma_{V} \stackrel{\text { def }}{=} L_{V} *_{q}-*_{q} L_{V}=0$ on any quotient forms. By using (6.2), we have $F_{A_{V}}=0$. Then by using Proposition 5.4 we get $d_{A}^{+s} d_{A}^{++s}=0$ for the $A$. In addition, by using (6.2), we have $d_{A}^{++s} d_{A}=l_{s} d_{A}^{++} d_{A}=l_{s}\left(\pi_{l} F_{A}+\pi_{q}^{+} F_{A}\right)=$ 0 .

It is not hard to compute $b_{+}^{j}(A, K)$ in this product case $M=N \times[0,1]$ with the pulled-back connection $A$ of the connection $A_{1}$ over $N$. To avoid repeating work we here quote the results from ( [10], Sections 7 and 8) directly.

If $K$ is empty, then $b=\pi_{l}$ on $\partial M$. By using ([10], Corollaries 8.6 and 8.11) for the pulled-back $A$ of the connection $A_{1}$, we have

$$
b^{j}(A)=b^{j}\left(A_{1}\right), \quad b_{+}^{m-1}(A)=b_{+}^{m-1}\left(A_{1}\right), \quad b_{+}^{m}(A)=b_{+}^{m}\left(A_{1}\right), \quad b_{+}^{m+1}(A)=0
$$

for $0 \leq j \leq m-2$. Therefore (4.9) yields

$$
\operatorname{ind}\left(T_{A}\right)_{\pi_{l}}=-\chi_{o_{q}}^{+}\left(A_{1}\right),
$$

where + refers to the $o_{q}$-orientation on $N \times\{1\}=N$, which is the same as the given orientation on $N$. Now (6.1) implies

$$
\operatorname{ind}\left(T_{A}\right)_{\pi_{l}}=\int_{N} a_{2 m}\left(T_{A}, \pi_{l}\right) \operatorname{vol}(N)+\int_{N^{-}} a_{2 m}\left(T_{A}, \pi_{l}\right) \operatorname{vol}\left(N^{-}\right) .
$$


Let $\phi$ be the isometry from $N \times\left(\frac{2}{3}, 1\right]$ to $N \times\left[0, \frac{1}{3}\right)$ by $(x, t) \rightarrow(x, 1-t)$ which preserves $\left(L, g, o_{q}, A\right)$. By using Proposition 6.2, we get

$$
\int_{N} a_{2 m}\left(T_{A}, \pi_{l}\right) \operatorname{vol}(N)=\int_{N^{-}} a_{2 m}\left(T_{A}, \pi_{l}\right) \operatorname{vol}\left(N^{-}\right)=\frac{1}{2} \operatorname{ind}\left(T_{A}\right)_{\pi_{l}}=-\frac{1}{2} \chi_{o_{q}}^{+}\left(A_{1}\right) .
$$

Now let $K=N \times\{1\}=N$. Thus $b=\pi_{q}$ on $N \times\{1\}=N$ and $b=\pi_{l}$ on $N \times\{0\}=N^{-}$. By using ([10], Corollaries 8.6 and 8.11), we get

$$
0=b^{j}(A, N)=b_{+}^{m-1}(A, N)=b_{+}^{m}(A, N)=b_{+}^{m+1}(A, N)
$$

for all $0 \leq j \leq m-2$. Thus

$$
\operatorname{ind}\left(T_{A}, \pi_{q}\right)=0 .
$$

By (4.9), (6.1) and (6.3), we get

$$
\int_{N} a_{2 m}\left(T_{A}, \pi_{q}\right) \operatorname{vol}(N)=-\int_{N^{-}} a_{2 m}\left(T_{A}, \pi_{l}\right) \operatorname{vol}\left(N^{-}\right)=-\frac{1}{2} \operatorname{ind}\left(T_{A}\right)_{\pi_{l}}=\frac{1}{2} \chi_{o_{q}}^{+}\left(A_{1}\right) .
$$

Now by using Proposition 6.2 and (6.1), (6.3) and (6.4), we have

$$
\operatorname{Ind}\left(T_{A}\right)_{b}=\frac{1}{2}\left[\chi_{o_{q}}^{+}\left(\left.A\right|_{K}\right)-\chi_{o_{q}}^{+}\left(\left.A\right|_{\partial M-K}\right)\right]
$$

in the case the restriction of $g$ to a collar of each boundary component of $M$ is isometric to the product metric $g_{0}$.

Because indices are deformation invariant, and because any two $L$-admissible metrics $g_{0}$ and $g_{1}$ can be deformed to each other by $L$-admissible metrics $t g_{0}+$ $(1-t) g_{1}$ and any two connections on the same bundle can be deformed to each other, we see that (6.5), which is the same as (2.25), holds for all data $\left(L, g, o_{q}\right)$ such that $g$ is $L$-admissible and any connection $A$ on $E$. Thus the proof of Theorem 2.9 is completed.

III. Proof of Theorem 2.6.

First let us recall briefly the definition of $\mathbb{C}-\mathbb{R}_{+}-\mathbb{R}_{-}$ellipticity of a first order operator with a zero-th order boundary operator from ([13], Section 1.9). Let $P$ be a first order formally self-adjoint elliptic differential operator $\Gamma(F) \rightarrow \Gamma(F)$, where $F$ is a complex vector bundle over a manifold $M$ with boundary $\partial M$ and $\Gamma(F)$ is the space of smooth sections of $F$. Let $\beta$ be a zero-th order boundary operator $\left.F\right|_{\partial M} \rightarrow F^{\prime}$ where $F^{\prime}$ is a complex vector bundle over $\partial M$ with $2 \operatorname{dim} F^{\prime}=\operatorname{dim} F$. Let $p(x, \xi)$ be the principal symbol of $P$ at $(x, \xi)$ where $\xi$ is a covector at $x \in M$. Near the boundary $\partial M, x=\left(x_{0}, x^{\prime}\right)$ where $x^{\prime}$ is a point in $\partial M$ and $x_{0}$ is the normal distance to the boundary $\partial M$, and $\xi=\left(\xi_{0}, \xi^{\prime}\right)$ according to the splitting $T^{*} M=T^{*} \mathbb{R} \oplus T^{*}(\partial M)$ induced by the identification of a neighborhood of $\partial M$ in $M$ with a collar $[0, \epsilon) \times \partial M$ for some $\epsilon>0$. This identification is induced by inward normal geodesic flows. Thus

$$
p(x, \xi)=p\left(x_{0}, x^{\prime}, \xi_{0}, \xi^{\prime}\right) .
$$

Consider the ordinary differential equation:

$$
p\left(0, x^{\prime}, \xi^{\prime},-i \frac{d}{d t}\right) f(t)=\lambda f(t), \quad \lim _{t \rightarrow \infty} f(t)=0,
$$

where $\left(\xi^{\prime}, \lambda\right) \neq(0,0) \in T^{*}(\partial M) \times\left(\mathbb{C}-\mathbb{R}_{+}-\mathbb{R}_{-}\right)$. The boundary problem $(P, \beta)$ is called $\mathbb{C}-\mathbb{R}_{+}-\mathbb{R}_{-}$elliptic if $(1) \operatorname{det}(p(x, \xi)-\lambda) \neq 0$ for all $x \in M-\partial M$ and 
$(\xi, \lambda) \neq(0,0) \in T^{*} M \times\left(\mathbb{C}-\mathbb{R}_{+}-\mathbb{R}_{-}\right)$, and (2) there is a unique solution $f(t)$ to the above ordinary differential equation such that $\beta f(0)=f^{\prime}$ for any given $f^{\prime} \in F^{\prime}$.

The symbol $p(x, \xi)$ can be expressed uniquely as follows.

$$
p(x, \xi)=p\left(x_{0}, x^{\prime}, \xi_{0}, \xi^{\prime}\right)=p_{0}\left(x^{\prime}\right) \xi_{0}+p\left(0, x^{\prime}, 0, \xi^{\prime}\right) .
$$

Define

$$
\tau\left(x^{\prime}, \xi^{\prime}, \lambda\right) \stackrel{\text { def }}{=} i p_{0}\left(x^{\prime}\right)^{-1}\left(p\left(x^{\prime}, 0, \xi^{\prime}, 0\right)-\lambda\right) .
$$

Lemma 6.4 ([13], Lemma 1.9.4). If $p(x, \xi)$ is self-adjoint and elliptic, then $\tau\left(x^{\prime}, \xi^{\prime}, \lambda\right)$ has no eigenvalue of zero real part for $\left(\xi^{\prime}, \lambda\right) \in T^{*}(\partial M) \times\left(\mathbb{C}-\mathbb{R}_{+}-\mathbb{R}_{-}\right)$ and $\left(\xi^{\prime}, \lambda\right) \neq(0,0)$.

Therefore, over $T^{*}(\partial M) \times\left(\mathbb{C}-\mathbb{R}_{+}-\mathbb{R}_{-}\right)$there is a direct sum decomposition $F=$ $F_{+}(\tau) \oplus F_{-}(\tau)$, where $F_{+}(\tau)\left(F_{-}(\tau)\right.$ resp. $)$ is fiberwisely spanned by eigenvectors of $\tau\left(x^{\prime}, \xi^{\prime}, \lambda\right)$ with eigenvalues of positive (negative resp.) real parts.

Lemma 6.5 ([13], Lemma 1.9.5). Suppose $P: \Gamma(F) \rightarrow \Gamma(F)$ is a first order formally self-adjoint elliptic differential operator with zero-th order boundary operator $\beta:\left.F\right|_{\partial M} \rightarrow F^{\prime}$. Then (1) $(P, \beta)$ is $\mathbb{C}-\mathbb{R}_{+}-\mathbb{R}_{-}$-elliptic if and only if $\beta: F_{+}(\tau) \rightarrow F^{\prime}$ is an isomorphism for $\left(\xi^{\prime}, \lambda\right) \neq(0,0) \in T^{*}(\partial M) \times\left(\mathbb{C}-\mathbb{R}_{+}-\mathbb{R}_{-}\right)$;

(2) $P_{\beta}$ is self-adjoint if and only if $p_{0}\left(x^{\prime}\right) \operatorname{Ker} \beta$ is perpendicular to Ker $\beta$ in $F$ for $x^{\prime} \in \partial M$.

Now we are going to study the symbol of the self-duality operator $T_{A} \oplus T_{A}^{*}$ in Theorem 2.6. Since $\mathbb{C}-\mathbb{R}_{+}-\mathbb{R}_{-}$ellipticity depends only on principal symbols, we need only to prove Theorem 2.6 for $d_{A}=d$. Recall that we use $T$ to denote $T_{A}$ for $d_{A}=d$.

Let the symbol of $T \oplus T^{*}$ at $(x, \xi)$ be denoted by $\sigma_{\xi}\left(T \oplus T^{*}\right)$, where $\xi$ is a covector at point $x$ in $M$. For the sake of brevity, let

$$
\xi \circ \stackrel{\text { def }}{=}-i \sigma_{\xi}\left(T \oplus T^{*}\right) .
$$

Recall that $T_{c}^{*} M \stackrel{\text { def }}{=} \mathbb{C} \otimes_{\mathbb{R}} T^{*} M$ and $Q_{c}^{*}=\mathbb{C} \otimes_{\mathbb{R}} Q^{*}$. When $m$ is odd, let $\bigwedge_{++}^{m} T_{c}^{*} M \stackrel{\text { def }}{=} L^{*} \wedge \bigwedge^{m-1} Q_{c}^{*} \oplus \bigwedge_{+}^{m} Q_{c}^{*}$ and let $\bigwedge^{m+1} T_{c}^{*} M \stackrel{\text { def }}{=} L^{*} \wedge \bigwedge_{+}^{m} Q_{c}^{*}$. When $m$ is even, let $\bigwedge_{++}^{m} T^{*} M \stackrel{\text { def }}{=} L^{*} \wedge \bigwedge^{m-1} Q^{*} \oplus \bigwedge_{+}^{m} Q^{*}$ and let $\bigwedge^{m+1} T^{*} M \stackrel{\text { def }}{=}$ $L^{*} \wedge \bigwedge_{+}^{m} Q^{*}$. Let

$F \stackrel{\text { def }}{=} \sum_{j=0}^{m-1} \bigwedge^{j} T_{c}^{*} M \oplus \bigwedge_{++}^{m} T_{c}^{*} M \oplus \bigwedge_{+}^{m+1} T_{c}^{*} M$ for odd $m$,

$F_{q} \stackrel{\text { def }}{=} \sum_{j=0}^{m-1} \bigwedge^{j} Q_{c}^{*} \oplus \bigwedge_{+}^{m} Q_{c}^{*}$ for odd $m$,

$F \stackrel{\text { def }}{=} \mathbb{C} \otimes_{\mathbb{R}}\left(\sum_{j=0}^{m-1} \bigwedge^{j} T^{*} M \oplus \bigwedge_{++}^{m} T^{*} M \oplus \wedge_{+}^{m+1} T^{*} M\right)$ for even $m$,

$F_{q} \stackrel{\text { def }}{=} \mathbb{C} \otimes_{\mathbb{R}}\left(\sum_{j=0}^{m-1} \bigwedge^{j} Q^{*} \oplus \bigwedge_{+}^{m} Q^{*}\right)$ for even $m$.

Then $F=L^{*} \wedge F_{q} \oplus F_{q}$ and $\xi \circ: F \rightarrow F$. Let $e^{0}$ be an oriented unit basis of $L$ over $M$ and let $\left(e^{1}, \ldots, e^{2 m}\right)$ be an $o_{q}$-oriented $g_{q}$-orthonormal basis of $\left.Q^{*}\right|_{U}$, where $U$ is an open neighborhood of $x$ in $M$. Let $J$ be an ordered subset of $\{1, \ldots, 2 m\}$ and $e^{J}$ be the wedge of $e^{j}$ in the order of $J$ where $j$ runs through $J$. Let $|J|$ be the length of $J$.

Proposition 6.6. Let $s$ be any nonzero complex number, $1 \leq j<k \leq 2 m$.

(1) When $\operatorname{dim} M=3$, let $e_{s}^{1} \stackrel{\text { def }}{=} \frac{\sqrt{2}}{|s|} e^{1}$ and $e_{s}^{2} \stackrel{\text { def }}{=} \frac{\sqrt{2}}{|s|} e^{2}$, then we have

$$
\left(e^{0} \circ\right)^{2}=-1, \quad\left(e_{s}^{1} \circ\right)^{2}=-1, \quad\left(e_{s}^{2} \circ\right)^{2}=-1,
$$


$e^{0} \circ e_{s}^{1} \circ+e_{s}^{1} \circ e^{0} \circ=0, \quad e^{0} \circ e_{s}^{2} \circ+e_{s}^{2} \circ e^{0} \circ=0, \quad e_{s}^{1} \circ e_{s}^{2} \circ+e_{s}^{2} \circ e_{s}^{1} \circ=0$.

(2) When $\operatorname{dim} M \geq 3$, we have

$$
\begin{gathered}
\left(e^{0} \circ\right)^{2}=-1, \quad e^{0} \circ e^{j} \circ+e^{j} \circ e^{0} \circ=0 \quad \text { for any nonzero } s \\
\left(e^{j} \circ\right)^{2}=-1, \quad e^{j} \circ e^{k} \circ+e^{k} \circ e^{j} \circ=0 \quad \text { if }|s|^{2}=2 .
\end{gathered}
$$

(3) When $\operatorname{dim} M \geq 5$, we have

$$
\left(e^{j} \circ\right)^{2}=-N_{j, s}^{2}, \quad e^{j} \circ e^{k} \circ+e^{k} \circ e^{j} \circ=C_{j k, s} \quad \text { for any nonzero } s .
$$

(4) For any nonzero $s$ and all $\operatorname{dim} M \geq 3$, we have that $e^{0} \circ=e^{0} \wedge$ on $F_{q}$, $e^{0} \circ=-i_{e^{0}}$ on $L^{*} \wedge F_{q}$, $e^{0} \circ$ interchanges $L^{*} \wedge F_{q}$ and $F_{q}$, and $e^{j} \circ$ preserves $L^{*} \wedge F_{q}$ and also preserves $F_{q}$ for all $1 \leq j \leq 2 m$.

This proposition will be proved at the end of this section. Note the above (3) means $\xi \circ$ is a representation of the Clifford multiplication when $|s|^{2}=2$.

$N_{j, s}$ and $C_{j k, s}$ are defined as follows. Let $m \geq 2$ and $0 \leq j \leq 2 m$. Define

$G_{j}=\left\langle e^{0} \wedge e^{J}, e^{J}:|J|=m-1\right.$ and $\left.j \notin J\right\rangle \oplus \bigwedge_{+}^{m} Q_{c}^{*}$ for odd $m$,

$G_{j}=\left\langle e^{0} \wedge e^{J}, e^{J}:|J|=m-1\right.$ and $\left.j \notin J\right\rangle \oplus \mathbb{C} \otimes_{\mathbb{R}} \wedge_{+}^{m} Q^{*}$ for even $m$.

Let $G_{j}^{\perp}$ be the orthogonal complement of $G_{j}$ in $\left.F\right|_{U}$. Define

$$
N_{j, s}=\frac{|s|}{\sqrt{2}} \text { on } G_{j}, \quad N_{j, s}=1 \text { on } G_{j}^{\perp} .
$$

Let $m \geq 2$ and $0 \leq j<k \leq 2 m$. Define

$G_{j k}=\left\langle e^{0} \wedge e^{j R}, e^{0} \wedge e^{k R}, e^{j R}, e^{k R}:|R|=m-2\right.$ and $\left.j, k \notin R\right\rangle$.

We define $C_{j k, s}=0$ on $G_{j k}^{\perp}$. On $G_{j k}$, we define $C_{j k, s}$ as the mapping:

$$
\begin{aligned}
& e^{j R} \rightarrow\left(-1+\frac{|s|^{2}}{2}\right) e^{k R}, \\
& e^{k R} \rightarrow\left(\frac{|s|^{2}}{2}-1\right) e^{j R}, \\
& e^{0} \wedge e^{j R} \rightarrow\left(-1+\frac{|s|^{2}}{2}\right) e^{0} \wedge e^{k R}, \\
& e^{0} \wedge e^{k R} \rightarrow\left(\frac{|s|^{2}}{2}-1\right) e^{0} \wedge e^{j R} .
\end{aligned}
$$

Lemma 6.7. (1) If $|s|^{2}=2$ then all $N_{j, s}=1$ and $C_{j k, s}=0$ on $\left.F\right|_{U}$ for $0 \leq j<$ $k \leq 2 m$.

(2) $N_{j, s}$ are positive definite real diagonal matrices with respect to the above basis for $G_{j} \oplus G_{j}^{\perp}=\left.F\right|_{U}$ for all $0 \leq j \leq 2 m$ and any nonzero $s$.

(3) $C_{j k, s}$ are real skew-symmetric matrices with respect to the above basis of $G_{j k} \oplus G_{j k}^{\perp}=\left.F\right|_{U}$ for all $0 \leq j<k \leq 2 m$ and any nonzero $s$.

Lemma 6.8. If $N$ is a positive definite Hermitian matrix and $C$ a skew-Hermitian matrix, then $\operatorname{det}(N+C) \neq 0$.

Proof. If $\operatorname{det}(N+C)=0$, then there is a vector $v \neq 0$ such that $N v=-C v$. Since $N$ is Hermitian and $C$ is skew-Hermitian, we have $\langle N v, v\rangle=\langle v, N v\rangle=-\langle v, C v\rangle=$ $\langle C v, v\rangle=-\langle N v, v\rangle$, and thus $\langle N v, v\rangle=0$, which contradicts the fact that $N$ is positive definite. 
By applying Proposition 6.6, we can follow the same arguments for the $\mathbb{C}-$ $\mathbb{R}_{+}-\mathbb{R}_{-}$ellipticity of De Rham operator $d+d^{*}$ with the classical relative and absolute boundary conditions. (See [13], pp.243-244.) In the following proof, when $\operatorname{dim} M=3$, let $e^{1}$ and $e^{2}$ be replaced respectively by $e_{s}^{1}$ and $e_{s}^{2}$ and let all $N_{j, s}=1$ and $C_{j k, s}=0$. Let $F^{\prime}$ be $F_{q}$ on $K$ and $L^{*} \wedge F_{q}$ on $\partial M-K$. Our $T \oplus T^{*}$ is obviously a first order formally self-adjoint operator. It has the symbol $p(x, \xi)=$ $i \xi \circ=\xi_{0} i e^{0} \circ+\sum_{j=1}^{2 m} \xi_{j} i e^{j} \circ$ where $\xi=\xi_{0} e^{0}+\sum_{j=1}^{2 m} \xi_{j} e^{j}$. Applying Proposition 6.6, we get

$$
p(x, \xi)^{2}=\xi_{0}^{2}+\sum_{j=1}^{2 m} \xi_{j}^{2} N_{j, s}^{2}+\sum_{j<k} \xi_{j} \xi_{k} C_{j k, s}
$$

where $0 \leq j, k \leq 2 m$. By Lemma $6.7, \xi_{0}^{2}+\sum_{j=1}^{2 m} \xi_{j}^{2} N_{j, s}^{2}$ is a positive definite Hermitian matrix for $\xi \neq 0$ and $\sum_{j<k} \xi_{j} \xi_{k} C_{j k, s}$ is a skew-Hermitian matrix. Thus by Lemma $6.8, \operatorname{det} p(x, \xi)^{2} \neq 0$ for all $\xi \neq 0$ and $T \oplus T^{*}$ is elliptic.

Let $\xi^{\prime}=\sum_{j=1}^{2 m} \xi_{j} e^{j}$. According to (6.6), we have

$$
\tau\left(x^{\prime}, \xi^{\prime}, \lambda\right)=i\left(i e^{0} \circ\right)^{-1}\left(\sum_{j=1}^{2 m} \xi_{j} i e^{j} \circ-\lambda\right)=-i \lambda q_{0}+\sum_{j=1}^{2 m} \xi_{j} q_{j},
$$

where $q_{0}=i e^{0}$ and $q_{j}=-i e^{0} \circ e^{j} \circ$ for $1 \leq j \leq 2 m$. Let $\tau$ denote $\tau\left(x^{\prime}, \xi^{\prime}, \lambda\right)$ in this proof, and let $\alpha=1$ on $L^{*} \wedge F_{q}$ and $\alpha=-1$ on $F_{q}$. Using (4) of Proposition 6.6, we see that all $q_{j}$ interchanges $L^{*} \wedge F_{q}$ and $F_{q}$. Thus $\alpha q_{j}=-q_{j} \alpha$ and $\alpha \tau=-\tau \alpha$. If $\mu$ is an eigenvalue for $\tau$ such that $\tau(\omega)=\mu \omega$ with $\omega \neq 0$, then $-\mu$ is also an eigenvalue for $\tau$, since $\tau(\alpha(\omega))=-\mu \alpha(\omega)$. Lemma 6.4 states that any eigenvalue of $\tau$ is not zero and has no zero real part. Thus $\operatorname{dim} F_{+}(\tau)=\operatorname{dim} F_{-}(\tau)$ and $\operatorname{dim} F_{+}(\tau)=\operatorname{dim} F^{\prime}=\frac{1}{2} \operatorname{dim} F$. Therefore we need only to prove $\left.b\right|_{F_{+}(\tau)}: F_{+}(\tau) \rightarrow$ $F^{\prime}$ is injective.

Since ker $b=L^{*} \wedge E_{q}$ over $K$ and $\operatorname{ker} b=F_{q}$ over $\partial M-K$, we have $\alpha= \pm 1$ on $\operatorname{ker} b$. If $\left.\operatorname{ker} b\right|_{F_{+}(\tau)}=\operatorname{ker} b \cap F_{+}(\tau)$ were not zero, then there were $\omega \neq 0$ such that $\alpha(\omega)= \pm \omega$ and $\tau(\omega)=\mu \omega$ for some $\mu \neq 0$. Using $\alpha \tau=-\tau \alpha$, we get $\mu \omega=-\mu \omega$ and $\mu=0$, which is a contradiction. This completes the $\mathbb{C}-\mathbb{R}_{+}-\mathbb{R}_{-}$ellipticity of $\left(T \oplus T^{*}, b\right)$ by Lemma 6.5. Directly using (2.14) and using Green's formula, one can easily see that $\left(T \oplus T^{*}, b\right)$ is self-adjoint. Thus the proof of Theorem 2.6 is completed.

IV. Proof of Proposition 6.6.

The case of $\operatorname{dim} M=3$ is the simplest case. Recall $e^{0}$ is an oriented unit basis of $L$ over $M$, and $\left(e^{1}, e^{2}\right)$ is an oriented $g_{q}$-orthonormal basis of $\left.Q^{*}\right|_{U}$ where $U$ is an open subset of $M$. Since $*_{q} e^{1}=e^{2}$ and $*_{q} e^{2}=-e^{1}, \bar{\delta}=e^{1}-i e^{2}$ is a complex basis for $\left.\bigwedge_{+}^{1} Q_{c}^{*}\right|_{U}$ and $\delta=e^{1}+i e^{2}$ is a complex basis for $\left.\bigwedge_{-}^{1} Q_{c}^{*}\right|_{U}$. Thus $e^{1}=\frac{\delta+\bar{\delta}}{2}$ and $e^{1}=\frac{\delta-\bar{\delta}}{2 i}$. Definition 2.3 applying to the case $m=1$ yields that

$$
\begin{gathered}
\xi \circ: \mathbb{C} \oplus \bigwedge_{++}^{1} T_{c}^{*} M \oplus \bigwedge_{+}^{2} T_{c}^{*} M \rightarrow \mathbb{C} \oplus \bigwedge_{++}^{1} T_{c}^{*} M \oplus \bigwedge_{+}^{2} T_{c}^{*} M, \\
\xi \circ\left(\omega_{0}+\omega_{1}+\omega_{2}\right)=-i_{\xi} l_{\bar{s}} \omega_{1}+l_{s} \pi^{++}\left(\xi \wedge \omega_{0}\right)-h_{\bar{s}} \pi^{++}\left(i_{\xi} \omega_{2}\right)+\pi^{+}\left(\xi \wedge h_{s} \omega_{1}\right) .
\end{gathered}
$$


$e^{0} \circ$ maps

$$
\begin{aligned}
& e^{0} \rightarrow-i_{e^{0}} l_{\bar{s}} e^{0}+\pi^{+}\left(e^{0} \wedge h_{s} e^{0}\right)=-1, \\
& \bar{\delta} \rightarrow-i_{e^{0}} l_{\bar{s}} \bar{\delta}+\pi^{+}\left(e^{0} \wedge h_{s} \bar{\delta}\right)=e^{0} \wedge \bar{\delta}, \\
& 1 \rightarrow l_{s} \pi^{++} e^{0}=e^{0} \\
& e^{0} \wedge \bar{\delta} \rightarrow-h_{\bar{s}} i_{e^{0}}\left(e^{0} \wedge \bar{\delta}\right)=-\bar{\delta} .
\end{aligned}
$$

$e^{1} \circ$ maps

$$
\begin{aligned}
& e^{0} \rightarrow-i_{e^{1}} l_{\bar{s}} e^{0}+\pi^{+}\left(e^{1} \wedge h_{s} e^{0}\right)=-\frac{s}{2} e^{0} \wedge \bar{\delta}, \\
& \bar{\delta} \rightarrow-i_{e^{1}} l_{\bar{s}} \bar{\delta}+\pi^{+}\left(e^{1} \wedge h_{s} \bar{\delta}\right)=-\bar{s}, \\
& 1 \rightarrow l_{s} \pi^{++} e^{1}=\frac{s}{2} \bar{\delta} \\
& e^{0} \wedge \bar{\delta} \rightarrow-h_{\bar{s}} i_{e^{1}}\left(e^{0} \wedge \bar{\delta}\right)=\bar{s} e^{0} .
\end{aligned}
$$

$e^{2} \circ$ maps

$$
\begin{aligned}
& e^{0} \rightarrow-i_{e^{2}} l_{\bar{s}} e^{0}+\pi^{+}\left(e^{2} \wedge h_{s} e^{0}\right)=\frac{s}{2 i} e^{0} \wedge \bar{\delta}, \\
& \bar{\delta} \rightarrow-i_{e^{2}} l_{\bar{s}} \bar{\delta}+\pi^{+}\left(e^{2} \wedge h_{s} \bar{\delta}\right)=\bar{s} i \\
& 1 \rightarrow l_{s} \pi^{++} e^{2}=-\frac{s}{2 i} \bar{\delta} \\
& e^{0} \wedge \bar{\delta} \rightarrow-h_{\bar{s}} i_{e^{2}}\left(e^{0} \wedge \bar{\delta}\right)=-i \bar{s} e^{0} .
\end{aligned}
$$

The claim (1) of Proposition 6.6 follows from the above tables. Now we prove claims (1)-(4) of Proposition 6.6 for $\operatorname{dim} M \geq 5$. For an ordered subset $J$ of $\{1, \ldots, 2 m\}$, let $*_{q} J$ be the ordered subset such that $J *_{q} J$ is an even permutation of ordered $\{1, \ldots, 2 m\}$. Let $\epsilon=1$ if $m$ is even and $\epsilon=-i$ if $m$ is odd in this proof. Thus $*_{q} e^{J}=e^{*_{q} J}$ and $\left\{\delta_{ \pm}^{J} \stackrel{\text { def }}{=} e^{1 J} \pm \epsilon e^{*_{q}(1 J)}\right.$ for $|J|=m-1$ and $\left.1 \notin J\right\}$ is an orthogonal basis of the same length for $\bigwedge_{ \pm}^{m} Q_{c}^{*}$ if $m$ is odd, and for $\mathbb{C} \otimes_{\mathbb{R}} \bigwedge_{ \pm}^{m} Q^{*}$ if $m$ is even. By Definition 2.3, we see that $\xi \circ: F \rightarrow F$ is given by

$$
\begin{gathered}
\xi \circ\left(\sum_{j=0}^{m-1} \omega_{j}+\omega_{m}+\omega_{m+1}\right)=\sum_{j=0}^{m-2} c(\xi)\left(\omega_{j-1}\right)+\xi \wedge \omega_{m-2}-i_{\xi} l_{\bar{s}} \omega_{m} \\
\quad+l_{s} \pi^{++}\left(\xi \wedge \omega_{m-1}\right)-h_{\bar{s}} \pi^{++}\left(i_{\xi} \omega_{m+1}\right)+\pi^{+}\left(\xi \wedge h_{s} \omega_{m}\right)
\end{gathered}
$$

where $c(\xi)=\xi \wedge-i_{\xi}$ is the usual Clifford multiplication. Thus $\xi \circ=c(\xi)$ on $\sum_{j=0}^{m-2} T_{c}^{*} M$.

We also have $\xi_{\circ}=c(\xi)$ on $L^{*} \wedge \bigwedge^{m-2} Q_{c}^{*}$. Indeed, let $\omega_{m-1} \in L^{*} \wedge \bigwedge^{m-2} Q^{*}$, then $\xi \wedge \omega_{m-1} \in L^{*} \wedge \wedge^{m-1} Q^{*}$ on which $l_{s} \pi^{++}$is identity. Thus $\xi \circ \omega_{m-1}=$ $l_{s} \pi^{++}\left(\xi \wedge \omega_{m-1}\right)-i_{\xi} \omega_{m-1}=\xi \wedge \omega_{m-1}-i_{\xi} \omega_{m-1}=c(\xi) \omega_{m-1}$.

Therefore we need only to prove Proposition 6.6 for covectors in $\bigwedge^{m-1} Q_{c}^{*} \oplus$ $\bigwedge_{++}^{m} T_{c}^{*} M \oplus \bigwedge_{+}^{m+1} T_{c}^{*} M$ when $m$ is odd and in $\bigwedge^{m-1} Q^{*} \oplus \bigwedge_{++}^{m} T^{*} M \oplus \bigwedge_{+}^{m+1} T^{*} M$ when $m$ is even. This space is $L^{*} \wedge H_{q} \oplus H_{q}$, where $H_{q} \stackrel{\text { def }}{=} \bigwedge^{m-1} Q_{c}^{*} \oplus \bigwedge_{+}^{m} Q_{c}^{*}$ when $m$ is odd, and $H_{q} \stackrel{\text { def }}{=} \bigwedge^{m-1} Q^{*} \oplus \bigwedge_{+}^{m} Q^{*}$ when $m$ is even.

In the following tables, $I, J, T, P, R, S$ are ordered subsets of $\{1, \ldots, 2 m\}$ such that

$|I|=m-1, \quad|J|=m-1$ and $1 \notin J, \quad|P|=m-1$ and $1,2 \notin P$, 
$|R|=m-2$ and $1 \notin R, \quad|S|=m-2$ and $1,2 \notin S, \quad|W|=m-3$ and $1,2 \notin W$.

When $|R|=0$ or $|S|=0$ in the case $m=2$, or $|W|=0$ in the case $m=3$, we regard $R, S$ and $W$ as empty sets and define $e^{e m p t y}=1$. When $|W|=-1$ in the case $m=2$, we delete the corresponding entry from the following tables. In addition, in the following, $L^{*}, H_{q}$ and $F$ means $\left.L^{*}\right|_{U},\left.H_{q}\right|_{U}$ and $\left.F\right|_{U}$ respectively.

On $L^{*} \wedge H_{q}, \quad e^{0} \circ \operatorname{maps}$

$$
\begin{aligned}
& e^{0} \wedge e^{I} \rightarrow \pi^{+}\left(e^{0} \wedge h_{s}\left(e^{0} \wedge e^{I}\right)\right)-i_{e^{0}} l_{\bar{s}}\left(e^{0} \wedge e^{I}\right)=-e^{I}, \\
& e^{0} \wedge \delta_{+}^{J} \rightarrow-h_{\bar{s}} \pi^{++} i_{e^{0}}\left(e^{0} \wedge \delta_{+}^{J}\right)=-\delta_{+}^{J} .
\end{aligned}
$$

On $H_{q}, \quad e^{0} \circ$ maps

$$
\begin{aligned}
& e^{I} \rightarrow l_{s} \pi^{++}\left(e^{0} \wedge e^{I}\right)-i_{e^{0}} e^{I}=e^{0} \wedge e^{I} \\
& \delta_{+}^{J} \rightarrow \pi^{+}\left(e^{0} \wedge h_{s} \delta_{+}^{J}\right)-i_{e^{0}} l_{\bar{s}} \delta_{+}^{J}=e^{0} \wedge \delta_{+}^{J} .
\end{aligned}
$$

On $L^{*} \wedge H_{q}, \quad e^{1} \circ \operatorname{maps}$

$$
\begin{aligned}
& e^{0} \wedge e^{1 R} \rightarrow \pi^{+}\left(e^{1} \wedge h_{s}\left(e^{0} \wedge e^{1 R}\right)\right)-i_{e^{1}} l_{\bar{s}}\left(e^{0} \wedge e^{1 R}\right)=e^{R}, \\
& e^{0} \wedge e^{J} \rightarrow \pi^{+}\left(e^{1} \wedge h_{s}\left(e^{0} \wedge e^{J}\right)\right)-i_{e^{1}} l_{\bar{s}}\left(e^{0} \wedge e^{J}\right)=-s \pi^{+}\left(e^{0} \wedge e^{1 J}\right)=\frac{-s}{2} e^{0} \wedge \delta_{+}^{J}, \\
& e^{0} \wedge \delta_{+}^{J} \rightarrow-h_{\bar{s}} \pi^{++} i_{e^{1}}\left(e^{0} \wedge \delta_{+}^{J}\right)=-h_{\bar{s}} \pi^{++}\left(-e^{0} \wedge e^{J}\right)=\bar{s} e^{0} \wedge e^{J} .
\end{aligned}
$$

On $H_{q}, \quad e^{1} \circ$ maps

$$
\begin{aligned}
& e^{1 R} \rightarrow l_{s} \pi^{++}\left(e^{1} \wedge e^{1 R}\right)-i_{e^{1}} e^{1 R}=-e^{R} \\
& e^{J} \rightarrow l_{s} \pi^{++}\left(e^{1} \wedge e^{J}\right)-i_{e^{1}} e^{J}=\frac{s}{2} \delta_{+}^{J} \\
& \delta_{+}^{J} \rightarrow \pi^{+}\left(e^{1} \wedge h_{s} \delta_{+}^{J}\right)-i_{e^{1}} l_{\bar{s}} \delta_{+}^{J}=\pi^{+}\left(e^{1} \wedge \epsilon e^{*_{q}(1 J)}\right)-\bar{s} \frac{1}{2} e^{J}=-\frac{\bar{s}}{2} e^{J} .
\end{aligned}
$$

The above tables imply $\left(e^{0} \circ\right)^{2}=-1, e^{0} \circ e^{1} \circ+e^{1} \circ e^{0} \circ=0$ and $\left(e^{1} \circ\right)^{2}=-N_{1, s}^{2}$ for any nonzero $s$. By $S O(2 m)$-symmetry of $Q^{*}$, we get $e^{0} \circ e^{j} \circ+e^{j} \circ e^{0} \circ=0$ and $\left(e^{j} \circ\right)^{2}=-N_{j, s}^{2}$ for any nonzero $s$.

On $H_{q}, \quad e^{1} \circ$ maps

$$
\begin{aligned}
& e^{12 W} \rightarrow l_{s} \pi^{++}\left(e^{1} \wedge e^{12 W}\right)-i_{e^{1}} e^{12 W}=-e^{2 W}, \\
& e^{1 S} \rightarrow l_{s} \pi^{++}\left(e^{1} \wedge e^{1 S}\right)-i_{e^{1}} e^{1 S}=-e^{S}, \\
& e^{2 S} \rightarrow l_{s} \pi^{++}\left(e^{1} \wedge e^{2 S}\right)-i_{e^{1}} e^{2 S}=\frac{s}{2} \delta_{+}^{2 S}, \\
& e^{P} \rightarrow l_{s} \pi^{++}\left(e^{1} \wedge e^{P}\right)-i_{e^{1}} e^{P}=\frac{s}{2} \delta_{+}^{P} \\
& \delta_{+}^{2 S} \rightarrow \pi^{+}\left(e^{1} \wedge h_{s} \delta_{+}^{2 S}\right)-i_{e^{1}} l_{\bar{s}} \delta_{+}^{2 S}=-\bar{s} e^{2 S}, \\
& \delta_{+}^{P} \rightarrow \pi^{+}\left(e^{1} \wedge h_{s} \delta_{+}^{P}\right)-i_{e^{1}} l_{\bar{s}} \delta_{+}^{P}=-\frac{\bar{s}}{2} e^{P} .
\end{aligned}
$$

In the following, $\left[*_{q}(1 P) / 2\right] \stackrel{\text { def }}{=} x$ such that $2 x=*_{q} 1 P$, that is, $e^{1 P 2 x}=$ $\operatorname{vol}\left(g_{q}\right)$, and $\left[*_{q}(2 P) / 1\right] \stackrel{\text { def }}{=} y$ such that $1 y=*_{q} 2 P$, that is, $e^{2 P 1 y}=\operatorname{vol}\left(g_{q}\right)$. We have $\left[*_{q}(1 P) / 2\right]=-\left[*_{q}(2 P) / 1\right]$ since $(-1)^{|P|} e^{12 P x}=e^{1 P 2 x}=\operatorname{vol}\left(g_{q}\right)=e^{2 P 1 y}=$ $(-1)^{|P|+1} e^{12 P y}$. 
On $H_{q}, \quad e^{2} \circ$ maps

$$
\begin{aligned}
& e^{12 W} \rightarrow l_{s} \pi^{++}\left(e^{2} \wedge e^{12 W}\right)-i_{e^{2}}\left(e^{1} \wedge e^{2 W}\right)=e^{1 W} \\
& e^{1 S} \rightarrow l_{s} \pi^{++}\left(e^{2} \wedge e^{1 S}\right)-i_{e^{2}} e^{1 S}=-\frac{s}{2} \delta_{+}^{2 S} \\
& e^{2 S} \rightarrow l_{s} \pi^{++}\left(e^{2} \wedge e^{2 S}\right)-i_{e^{2}} e^{2 S}=-e^{S} \\
& e^{P} \rightarrow l_{s} \pi^{++}\left(e^{2} \wedge e^{P}\right)-i_{e^{2}} e^{P}=s \frac{e^{2 P}+\epsilon e^{*_{q}(2 P)}}{2} \\
& =s \epsilon \frac{e^{*_{q}(2 P)}+\epsilon e^{*_{q} *_{q}(2 P)}}{2}=s \epsilon \delta^{\left[*_{q}(2 P) / 1\right]}, \\
& \delta_{+}^{2 S} \rightarrow \pi^{+}\left(e^{2} \wedge h_{s} \delta_{+}^{2 S}\right)-i_{e^{2}} l_{\bar{s}} \delta_{+}^{2 S}=\pi^{+}\left(e^{1} \wedge \epsilon e^{*_{q}(12 R)}\right)-\bar{s} \frac{1}{2} e^{2 S}=-\bar{s} e^{1 S}, \\
& \delta_{+}^{P} \rightarrow \pi^{+}\left(e^{2} \wedge h_{s} \delta_{+}^{P}\right)-i_{e^{2}} l_{\bar{s}} \delta_{+}^{P}=-\bar{s} \epsilon i_{e^{2}} \frac{e^{1 P}+\epsilon e^{*_{q}(1 P)}}{2}=-\bar{s} \epsilon e^{\left[*_{q}(1 P) / 2\right]} .
\end{aligned}
$$

On $H_{q}, \quad e^{1} \circ e^{2} \circ$ maps

$$
\begin{aligned}
& e^{12 W} \rightarrow-e^{2 W} \rightarrow e^{W}, \\
& e^{1 S} \rightarrow-e^{S} \rightarrow-e^{2 S} \\
& e^{2 S} \rightarrow \frac{s}{2} \delta_{+}^{2 S} \rightarrow \frac{1}{2}|s|^{2} e^{1 S}, \\
& e^{P} \rightarrow \frac{s}{2} \delta_{+}^{P} \rightarrow-\frac{1}{2}|s|^{2} \epsilon e^{\left[*_{q}(1 P) / 2\right]}, \\
& \delta_{+}^{2 S} \rightarrow \bar{s} e^{2 S} \rightarrow \bar{s} e^{S} \\
& \delta_{+}^{P} \rightarrow-\frac{1}{2} \bar{s} e^{P} \rightarrow-\frac{1}{2}|s|^{2} \epsilon \delta^{\left[*_{q}(2 P) / 1\right]} .
\end{aligned}
$$

On $H_{q}, \quad e^{2} \circ e^{1} \circ$ maps

$$
\begin{aligned}
& e^{12 W} \rightarrow e^{1 W} \rightarrow-e^{W} \\
& e^{1 S} \rightarrow-\frac{s}{2} \delta_{+}^{2 S} \rightarrow \frac{1}{2}|s|^{2} e^{2 S} \\
& e^{2 S} \rightarrow-e^{S} \rightarrow-e^{1 S}, \\
& e^{P} \rightarrow s \epsilon \delta^{\left[*_{q}(2 P) / 1\right]} \rightarrow-\frac{1}{2}|s|^{2} \epsilon e^{\left[*_{q}(2 P) / 1\right]}, \\
& \delta_{+}^{2 S} \rightarrow \bar{s} e^{1 S} \rightarrow-\bar{s} e^{S}, \\
& \delta_{+}^{P} \rightarrow-\frac{1}{2} \bar{s} \epsilon e^{\left[*_{q}(1 P) / 2\right]} \rightarrow-\frac{1}{2}|s|^{2} \epsilon \delta^{\left[*_{q}(1 P) / 2\right]} .
\end{aligned}
$$

Recall $e^{0} \circ=e^{0} \wedge$ on $H_{q} \subset F_{q}$ and $e^{0} \circ e^{j} \circ=-e^{j} \circ e^{0}$ for all $0 \leq j \leq 2 m$. Thus the above tables for $e^{1} \circ e^{2} \circ$ and $e^{1} \circ e^{2} \circ$ on $H_{q}$ also give the corresponding tables for $e^{1} \circ e^{2} \circ$ and $e^{1} \circ e^{2} \circ$ on $L^{*} \wedge H_{q}$. By these tables, we have $e^{1} \circ e^{2} \circ+e^{2} \circ e^{1}=C_{12, s}$ on $F$. By $S O(2 m)$-symmetry of $Q^{*}$ we get $e^{j} \circ e^{k} \circ+e^{k} \circ e^{j}=C_{j k, s}$ on $F$ for $0 \leq j<k \leq 2 m$. The proof of Proposition 6.6 is completed.

\section{REFERENCES}

[1] V.I.Arnold, Problems on singularity and dynamical system, preprint, 1992.

[2] M.F.Atiyah, Vector fields on manifolds, Arbeitsgemeinschaft für Forschung des Landes Nordrhein-Westfalen, 200, 1970. MR 41:7707 
[3] M.F.Atiyah, The index theorem for manifolds with boundary, Appendix I in seminar on the Atiyah-Singer index theorem, Ann. Math. Stud. 57(1965), Princeton Univ. Press, 337-351. MR 33:6649

[4] M.F.Atiyah and R.Bott, The index theorem for manifolds with boundary, Differential Analysis (papers collected at the Bombay Colloquium 1964), Oxford Univ. Press, 175-186. MR 32:3069

[5] M.F.Atiyah, N.J. Hitchin, and I.M.Singer, Self-duality in four dimensional Riemannian geometry, Proc. Royal Soc. London, Ser.A 362, (1978). 425-461. MR 80d:53023

[6] D.E.Blair, Contact manifolds in Riemannian geometry, Springer-Verlag, 1976. MR 57:7444

[7] M.Brunella and E.Ghys, Umbilical foliations and transversely holomorphic flows, J. Diff. Geom. 41(1995), 1-19. MR 95k:53039

[8] S.K.Donaldson, Irrationality and the h-cobordism conjecture, J. Diff. Geom. 26(1986), 141168. MR 88j:57035

[9] S.K.Donaldson and P.B.Kronheimer, The geometry of four-manifolds, Oxford Science Publications, Oxford, 1990. MR 92a:57036

[10] H.H.Fan, Half De Rham complexes and line fields on odd dimensional manifolds, Trans. Amer. Math. Soc. 348(1996), no.8, 2947-2982. MR 96k:58204

[11] H.H.Fan, Gauge fields and line fields on five-manifolds, preprint, Yale, 1995.

[12] H.H.Fan, Seiberg-Witten-type equation on five-manifolds, preprint, Yale, 1995.

[13] P.B.Gilkey, Invariance theory, the heat equation, and the Atiyah-Singer index theorem, Publish or Perish, 1984. MR 86j:58144

[14] M.W.Hirsch, Differential topology, Springer-Verlag, 1976. MR 56:6669

[15] P.B.Kronheimer and T.S.Mrowka, The genus of embedded surfaces in the projective plane, Math. Res. Letters 1, 797-808 (1994) MR 96a:57073

[16] P.B.Kronheimer and T.S.Mrowka, Embedded surfaces and the structure of Donaldson's Polynomial Invariants, J. Diff. Geom. 3 (1995) 573-734. MR 96e:57019

[17] P.Molino, Riemannian foliations, Birkhäuser, Boston, 1988 MR 89b:53054

[18] J.W.Morgan, Z.Szabó and C.H.Taubes. The generalized Thom conjecture, preprint, 1995.

[19] D.B.Ray and I.M.Singer, R-torsion and the Laplacian on Riemannian manifolds, Advan. Math. 7 (1971), 145-210. MR 45:4447

[20] C.H.Taubes, The Seiberg-Witten invariants and symplectic forms, Math. Res. Letters 1, 809-822 (1994). MR 95j:57039

[21] I.Vaisman, Conformal foliations, Kodai Math. J. 2 (1979), 26-37. MR 80g:57038

Department of Mathematics, Yale University, New Haven, Connecticut 06520

E-mail address: hhfan@math.yale.edu 